\title{
ON THE COSMIC EVOLUTION OF THE SCALING RELATIONS BETWEEN BLACK HOLES AND THEIR HOST GALAXIES: BROAD-LINE ACTIVE GALACTIC NUCLEI IN THE zCOSMOS SURVEY*
}

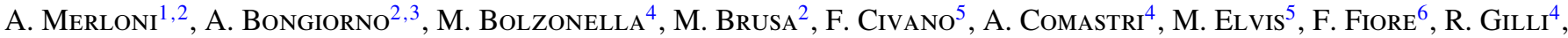

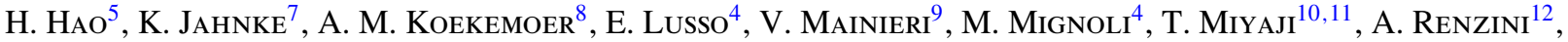 \\ M. Salvato ${ }^{13,14}$, J. Silverman ${ }^{15}$, J. TRump ${ }^{16}$, C. Vignali ${ }^{17}$, G. Zamorani ${ }^{4}$, P. Capak ${ }^{13,18}$, S. J. Lilli ${ }^{15}$, D. Sanders ${ }^{19}$,

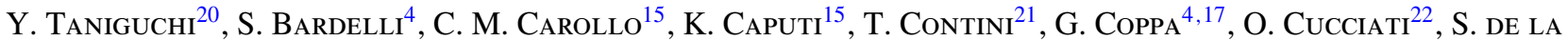 \\ Torre $^{22,23,24}$, L. De Ravel ${ }^{22}$, P. Franzetti ${ }^{23}$, B. Garilli ${ }^{23}$, G. Hasinger ${ }^{2,14}$, C. ImPey $^{16}$, A. Iovino ${ }^{23}$, K. Iwasawa ${ }^{4}$, \\ P. KampCZyK ${ }^{15}$, J.-P. KNeIr ${ }^{22}$, C. KNOBeL ${ }^{15}$, K. KovaČ ${ }^{15}$, F. Lamareille ${ }^{21}$, J. -F. Le Borgne ${ }^{21}$, V. Le Brun ${ }^{22}$, O. Le Fèvre ${ }^{22}$,

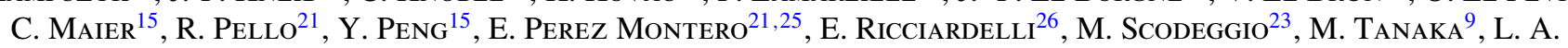 \\ M. TASCA ${ }^{22,23}$, L. TRESSE $^{22}$, D. Vergani ${ }^{4}$, AND E. ZuCCA ${ }^{4}$ \\ ${ }^{1}$ Excellence Cluster Universe, TUM, Boltzmannstr. 2, 85748, Garching, Germany \\ 2 Max Planck Institut für Extraterrestrische Physik, Giessenbachstr., 85471 Garching, Germany \\ ${ }^{3}$ University of Maryland, Baltimore County, 1000 Hilltop Circle, Baltimore, MD21250, USA \\ ${ }^{4}$ INAF-Osservatorio Astronomico di Bologna, via Ranzani 1, I-40127 Bologna, Italy \\ ${ }^{5}$ Harvard-Smithsonian Center for Astrophysics, 60 Garden Street, Cambridge, MA 02138, USA \\ ${ }^{6}$ INAF-Osservatorio Astronomico di Roma, via Frascati 33, Monteporzio (Rm), I00040, Italy \\ ${ }^{7}$ Max Planck Institut für Astronomie, Königstuhl 17, Heidelberg, D-69117, Germany \\ ${ }^{8}$ Space Telescope Science Institute, 3700 San Martin Drive, Baltimore, MD 21218, USA \\ ${ }^{9}$ ESO, Karl-Schwarzschild-Strasse 2, D-85748 Garching, Germany \\ ${ }^{10}$ Instituto de Astronomia, UNAM-Ensenada, Km 103 Carretera Tijuana-Ensenada, 22860 Ensenada, BC Mexico, USA \\ ${ }^{11}$ Center for Astrophysics and Space Sciences, University of California at San Diego, Code 0424, 9500 Gilman Drive, La Jolla, CA 92093, USA \\ 12 INAF - Osservatorio Astronomico di Padova, Padova, Italy \\ 13 California Institute of Technology, MC 105-24, 1200 East California Boulevard, Pasadena, CA 91125, USA \\ ${ }^{14}$ Max-Planck-Institute für Plasmaphysik, Boltzmannstrasse 2, D-85748 Garching, Germany \\ 15 Department of Physics, ETH Zurich, CH-8093 Zurich, Switzerland \\ ${ }^{16}$ Steward Observatory, University of Arizona, 933 North Cherry Avenue, Tucson, AZ 85721, USA \\ ${ }^{17}$ Dipartimento di Astronomia, Universitá di Bologna, via Ranzani 1, I-40127, Bologna, Italy \\ ${ }^{18}$ Spitzer Science Center, 314-6 Caltech, Pasadena, CA 91125, USA \\ ${ }^{19}$ Institute for Astronomy, University of Hawaii, 2680 Woodlawn Drive, HI 96822, USA \\ ${ }^{20}$ Research Centre for Space and Cosmic Evolution, Ehime University, Bunkyo-cho 2-5, Matsuyama 790-8577, Japan \\ ${ }^{21}$ Laboratoire d'Astrophysique de Toulouse-Tarbes, Université de Toulouse, CNRS, 14 avenue Edouard Belin, F-31400 Toulouse, France \\ ${ }^{22}$ Laboratoire d'Astrophysique de Marseille, CNRS-Univeristé d'Aix-Marseille, 38 rue Frederic Joliot Curie, 13388 Marseille Cedex 13, France \\ ${ }^{23}$ INAF-IASF, Via Bassini 15, I-20133, Milano, Italy \\ 24 INAF Osservatorio Astronomico di Brera, Via Brera 28, I-20121 Milano, Italy \\ 25 Instituto de Astrofisica de Andalucia, CSIC, Apdo. 3004, 18080, Granada, Spain \\ ${ }^{26}$ Dipartimento di Astronomia, Universitá di Padova, vicolo Osservatorio 3, I-35122 Padova, Italy \\ Received 2009 July 14; accepted 2009 October 30; published 2009 December 9
}

\begin{abstract}
We report on the measurement of the physical properties (rest-frame $K$-band luminosity and total stellar mass) of the hosts of 89 broad-line (type-1) active galactic nuclei (AGNs) detected in the zCOSMOS survey in the redshift range $1<z<2.2$. The unprecedented multi-wavelength coverage of the survey field allows us to disentangle the emission of the host galaxy from that of the nuclear black hole in their spectral energy distributions (SEDs). We derive an estimate of black hole masses through the analysis of the broad Mg II emission lines observed in the mediumresolution spectra taken with VIMOS/VLT as part of the zCOSMOS project. We found that, as compared to the local value, the average black hole to host-galaxy mass ratio appears to evolve positively with redshift, with a best-fit

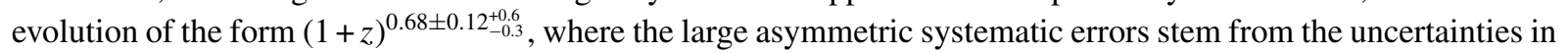
the choice of initial mass function, in the calibration of the virial relation used to estimate BH masses and in the mean QSO SED adopted. On the other hand, if we consider the observed rest-frame $K$-band luminosity, objects tend to be brighter, for a given black hole mass, than those on the local $M_{\mathrm{BH}}-M_{K}$ relation. This fact, together with more indirect evidence from the SED fitting itself, suggests that the AGN hosts are likely actively star-forming galaxies. A thorough analysis of observational biases induced by intrinsic scatter in the scaling relations reinforces the conclusion that an evolution of the $M_{\mathrm{BH}}-M_{*}$ relation must ensue for actively growing black holes at early times: either its overall normalization, or its intrinsic scatter (or both) appear to increase with redshift. This can be interpreted as signature of either a more rapid growth of supermassive black holes at high redshift, a change of structural properties of AGN hosts at earlier times, or a significant mismatch between the typical growth times of nuclear black holes and host galaxies. In any case, our results provide important clues on the nature of the early co-evolution of black holes and galaxies and challenging tests for models of AGN feedback and self-regulated growth of structures.
\end{abstract}

Key words: cosmology: observations - galaxies: active - galaxies: evolution - quasars: emission lines

Online-only material: color figures 


\section{INTRODUCTION}

Tight scaling relations between the central black holes mass and various properties of their host spheroids (velocity dispersion, $\sigma_{*}$, stellar mass, $M_{*}$, luminosity, core mass deficit) characterize the structure of nearby inactive galaxies (Magorrian et al. 1998; Gebhardt et al. 2000; Ferrarese \& Merritt 2000; Tremaine et al. 2002; Marconi \& Hunt 2003; Häring \& Rix 2004; Graham 2004; Hopkins, et al. 2007a; Kormendy \& Bender 2009; Gültekin et al. 2009). A result of the search for local QSO relics via the study of their dynamical influence on the surrounding stars and gas made possible by the launch of the Hubble Space Telescope (HST) nearly 20 years ago, these correlations have revolutionized the way we conceive the physical link between galaxy and AGN evolution. Coupled with the fact that supermassive black holes (SMBHs) growth is now known to be due mainly to radiatively efficient accretion over cosmological times, taking place during "active" phases (Sołtan 1982; Marconi et al. 2004; Shankar et al. 2004; Merloni \& Heinz 2008), this led to the suggestion that most, if not all, galaxies went through a phase of nuclear activity in the past, during which a strong physical coupling (generally termed "feedback") must have established a long-lasting link between host's and black hole's properties.

This shift of paradigm has sparked the activity of theoretical modelers. Following pioneering analytic works (Ciotti \& Ostriker 1997, 2001; Silk \& Rees 1998; Fabian 1999; Cavaliere \& Vittorini 2002; Wyithe \& Loeb 2003), widely different approaches have been taken to study the role of the AGN in galaxy evolution. Semi-analytic models (SAMs) have been the most numerous (Monaco et al. 2000; Kauffmann \& Haehnelt 2000; Volonteri et al. 2003; Granato et al. 2004; Menci et al. 2006; Croton et al. 2006; Bower et al. 2006; Malbon et al. 2007; Marulli et al. 2008; Somerville et al. 2008), and, among other things, have helped establishing the importance of latetime feedback from the radio-active AGN for the high-mass end of the galaxy mass function. However, firmer conclusions on the physical nature of such a feedback mode have been hampered by the large freedom SAM have in choosing baryonic physics recipes to implement in their schemes. On the other hand, fully hydrodynamic simulations of the cosmological evolution of SMBH have been also performed (Di Matteo et al. 2003, 2008; Sijacki et al. 2007; Coldberg \& Di Matteo 2008), but their computational costs have so far allowed only a limited exploration of sub-grid prescriptions (AGN physical models) in relatively small cosmological volumes. A third, hybrid, approach has also been followed, in which the results of high-resolution hydrodynamic simulations of galaxy-galaxy mergers with black holes (Di Matteo et al. 2005; Springel et al.

\footnotetext{
* Based on observations obtained at the European Southern Observatory (ESO) Very Large Telescope (VLT), Paranal, Chile, as part of the Large Program 175.A-0839 (the zCOSMOS Spectroscopic Redshift Survey). Also based on observations with the NASA/ESA Hubble Space Telescope, obtained at the Space Telescope Science Institute, which is operated by AURA Inc., under NASA contract NAS 5-26555; and on data collected at the Subaru Telescope, which is operated by the National Astronomical Observatory of Japan; the XMM-Newton, an ESA science mission with instruments and contributions directly funded by ESA Member States and NASA; the European Southern Observatory under Large Program 175.A-0839, Chile; the National Radio Astronomy Observatory which is a facility of the National Science Foundation operated under cooperative agreement by Associated Universities, Inc.; and the Canada-France-Hawaii Telescope with MegaPrime/MegaCam operated as a joint project by the CFHT Corporation, CEA/DAPNIA, the National Research Council of Canada, the Canadian Astronomy Data Centre, the Centre National de la Recherche Scientifique de France, TERAPIX, and the University of Hawaii.
}

2005) have been used to construct a general framework for merger-induced AGN feedback, capable of passing numerous observational tests (Hopkins et al. 2006a). Almost all the approaches outlined above use the local scaling relations as a constraint to the model parameters. As such, these relations have proved themselves unable to unambiguously determine the physical nature of the SMBH-galaxy coupling. One obvious way out of this impasse is to study their redshift evolution that different models predict to be different (Granato et al. 2004; Robertson et al. 2006; Croton 2006; Fontanot et al. 2006; Malbon et al. 2007; Marulli et al. 2008; Hopkins et al. 2009).

From the observational point of view, the current situation is far from being clear. In recent years, a number of groups have employed different techniques to try and detect signs of evolution in any of the locally observed scaling relations. Most efforts have been devoted to the study of the $M_{\mathrm{BH}}-\sigma_{*}$ relation. Shields et al. (2003) and Salviander et al. (2007) have used narrow nebular emission lines ([O III], [O II $]$ ) excited by the AGN emission in the nuclear region of galaxies as proxies for the central velocity dispersion, and compared these to the black hole mass estimated from the broad line width of QSOs from $z \sim 0$ to $z \sim 3$ (see Section 4). In both cases, a large scatter has been found in the relation between $M_{\mathrm{BH}}$ and $\sigma_{*}$, and the results are either in favor of (Salviander et al. 2007) or against (Shields et al. 2003) a positive evolution of the black hole mass to host dispersion ratio. However, as pointed out by Botte et al. (2004) and Greene \& Ho (2005), there are a number of problems with the underlying assumption that the narrow emission lines are good probes of the central gravitational potential, and the systematic uncertainties this method is endowed with are large. Komossa \& Xu (2007) have also shown that the AGN for which the [O III] emission line width is far broader than the host galaxy's stellar velocity dispersion $\sigma_{*}$ tend to show clear sign of blueshift in the narrow emission line (of the order of $100 \mathrm{~km} \mathrm{~s}^{-1}$ or larger). Thus, using [O III] as a proxy for $\sigma_{*}$ would at least require good enough spectral resolution to measure such blueshifts.

An alternative path has been followed by Woo et al. (2006), Treu et al. (2007), and Woo et al. (2008), who have studied carefully samples of moderately bright AGNs in narrow redshift ranges $(z \sim 0.36$ and 0.57$)$, where the host's stellar velocity dispersion can be measured directly from the absorption lines in high signal-to-noise spectra. They also found evidence of (strong) positive evolution of the $M_{\mathrm{BH}}$ to $\sigma_{*}$ ratio compared to the local value. This method, although promising and reliable, is quite inefficient and telescope-time consuming: secure detection of spectral absorption features in massive ellipticals at $1 \lesssim z \lesssim$ 2 require hundreds of hours of integration time on a $8 \mathrm{~m}$ class telescope (Cimatti et al. 2008).

Other groups have chosen to try and derive information on the host mass of the broad-line AGN (BLAGN) using multi-color image decomposition techniques (Jahnke et al. 2004; Sanchez et al. 2004) or spatially deconvolving optical spectra (Letawe et al. 2007). Due to the severe surface brightness dimming effects, employing these techniques for high redshift QSOs becomes increasingly challenging (but see Schramm et al. 2008; Jahnke et al. 2009; Decarli et al. 2009), unless gravitationally lensed QSOs are selected (Peng et al. 2006b; Ross et al. 2009). In all cases, very deep, high-resolution optical images (HST) are necessary to reliably disentangle the nuclear from the host-galaxy emission. Statistically, the most significant results have been published by Peng et al. (2006b), who, based on a large sample of 51 AGNs (both lensed and nonlensed) in the range $1<z<4.5$, observed that the ratio $M_{\mathrm{BH}} / M_{*}$ increases 
with look-back time, up to a factor $\simeq 4_{-1}^{+2}$ at the highest redshift probed.

Also cold/molecular gas motions on large galactic scales have been used to infer the properties (total mass in particular) of the hosts of AGNs at different redshifts. In the local universe, $\mathrm{H}_{\mathrm{I}}$ $21 \mathrm{~cm}$ lines have been used by Ho et al. (2008) to derive total stellar masses of the bulges around Seyfert-like AGNs. Using instead CO lines to estimate the velocity dispersion in highredshift QSO hosts, Walter et al. (2004) and Shields et al. (2006) find tentative evidence that the hosts are very undermassive compared to their central BHs (i.e., a positive redshift evolution of the $M_{\mathrm{BH}} / M_{*}$ ratio), a result confirmed by the study of Ho (2007).

Finally, a completely different approach has been that of trying to follow and compare the evolution of global descriptors of the galaxy and SMBH populations, such as mass densities (Merloni et al. 2004; Hopkins et al. 2006b; Shankar et al. 2009). Using the simple ansatz that total black hole mass density can only increase with time, and requiring that the limits imposed by local demographics be not violated, these works showed in general very moderate, if any, signs of cosmological evolution of the average black hole to host mass ratio.

Here we present a new method to tackle the issue of studying black hole-galaxy scaling relations at high redshift. Starting from a sample of the un-obscured AGN, for which the broad line kinematics can be used to infer the central SMBH mass, we take advantage of the unprecedentedly deep multiwavelength coverage of the COSMOS (Scoville et al. 2007) field and develop a novel spectral energy distribution (SED) fitting technique that allows us to decompose the entire SED into a nuclear AGN and host-galaxy components (Bongiorno et al. 2007). We show here how, for the majority of the objects in our sample, rest-frame $K$-band luminosity and total stellar mass of the host can be robustly determined, opening the way to a detailed study of the scaling relations in type-1 AGNs at $1 \lesssim z \lesssim 2.2$

The structure of the paper is the following: we will begin (Section 2) by introducing our sample, before proceeding to a discussion of our SED fitting method in Section 3, focusing our attention on the measures of the rest-frame $K$-band luminosity of the AGN hosts (Section 3.1), on their total stellar mass (Section 3.2). In Section 4, we will describe our estimates of the black hole masses obtained by studying the properties of the broad Mg II emission line, while in Section 4.3 we will briefly outline the characteristics of our AGN sample in terms of bolometric luminosity, BH mass, and accretion rates. Section 5 contains the main novel results of our study, namely the analysis of the scaling relation for the objects in our sample, as well as a characterization of their observed redshift evolution. An important part of our analysis, however, is the assessment of possible observational biases responsible for the trend observed, that we carry out in Section 6. This allows us to reach robust conclusions, that we discuss at the end of the paper, in Section 7.

Throughout this paper, we use the standard cosmology $\left(\Omega_{m}=\right.$ $0.3, \Omega_{\Lambda}=0.7$, with $H_{0}=70 \mathrm{~km} \mathrm{~s}^{-1} \mathrm{Mpc}^{-1}$ ).

\section{THE zCOSMOS Type-1 AGN SAMPLE}

Our AGN sample consists of the subsample of objects in the zCOSMOS bright spectroscopic catalog (Lilly et al. 2007) for which one or more broad emission lines have been identified in the spectrum. As such, it will be in the following identified as either a BLAGN or a type-1 AGN sample, without introducing any distinction between the two terms.
The zCOSMOS bright sample consists, at the times of writing, of 10,644 (medium resolution, MR) spectra observed with the VIMOS multi-object spectrograph on ESO-VLT in the COSMOS field, selected only on the basis of their $I_{A B}$ magnitude $\left(I_{A B}<22.5\right)$, based on the HST/Advanced Camera for Surveys (ACS) imaging of the COSMOS field (Koekemoer et al. 2007). For a detailed description of the spectroscopic survey we refer the reader to Lilly et al. $(2007,2009)$.

Within the zCOSMOS database, we have selected objects with broad emission lines full width half maximum (FWHM) larger than $2000 \mathrm{~km} \mathrm{~s}^{-1}$, a secure threshold for truly broadened lines, as compared to our spectral resolution $(R \sim 580$ for the MR grism, corresponding to $\sim 4.8 \AA$ and $\sim 520 \mathrm{~km} \mathrm{~s}^{-1}$ at the wavelength of $\mathrm{Mg}$ II emission). The final sample of type-1 AGN spectra selected from the zCOSMOS survey consists of 164 objects which correspond to about $1.8 \%$ of the objects in the total zCOSMOS database with measured redshift.

We measure black hole masses by applying the "virial" or "empirically calibrated photo-ionization" method (Wandel et al. 1999; Kaspi et al. 2000; Peterson et al. 2004; Bentz et al. $2006)$ to the $\mathrm{Mg}$ II $\lambda 2798$ broad emission line. We have selected BLAGNs in the redshift interval $z \sim[1.06,2.19]$, within which Mg II $\lambda 2798$ can be measured reliably. Taking into account the expected broad line width and the problems occurring at the edge of the spectrograph (e.g., fringing in the red part of the spectrum) we estimate that the spectral range available to measure $\mathrm{Mg}$ II line widths is $\sim 5650-9150 \AA$ (VIMOS spectral wavelengths range from $5500 \AA$ to $9500 \AA$ ).

Within this range, the zCOSMOS bright sample contains 104 AGNs. After a quick inspection, 15 of them have been excluded from the analysis because of the low quality of the available spectra, leaving us with 89 objects. Ten of those have radio counterparts (at $1.4 \mathrm{GHz}$ ) in the VLA/COSMOS catalogs (Schinnerer et al. 2007; Bondi et al. 2008), and are listed in Table 2.

\subsection{Photometry}

The zCOSMOS BLAGN sample has been cross correlated with the optical multi-band catalog of Capak et al. (2007), the CFHT / $K$ band catalog of Mc Cracken et al. (2009), the Infrared Array Camera (IRAC) catalog by Sanders et al. (2007), and the MIPS catalog by Le Floch et al. (2009). Briefly, the optical catalog contains about 3 million objects detected in at least one of the Subaru bands $(b, v, g, r, i, z)$ down to a $\mathrm{AB}$ magnitude limit of $\sim 27$ (see Capak et al. 2007; Taniguchi et al. 2007 for more details). From this catalog, it is possible to extract a subsample of $\sim 1.3$ million sources which have signal to noise $>5$ in the $i$ - or $z$-bands. The $K$-band catalog contains about $5 \times 10^{5}$ galaxies detected at a $\mathrm{S} / \mathrm{N}>5$ down to $\mathrm{K}(\mathrm{AB})=23.5$ (Mc Cracken et al. 2009). The IRAC catalog contains about $4 \times 10^{5}$ objects detected in the $3.6 \mu \mathrm{m}$ (IRAC channel 1) band and it is $90 \%$ complete at $>1 \mu \mathrm{Jy}(\mathrm{AB}=23.9)$. For each source in the catalog, the photometry from all the other IRAC channels is also reported. The MIPS catalog, obtained in Cycles 2, 3, and 4 , has very accurate photometry (Sanders et al. 2007). As described in details in Salvato et al. (2009), the fluxes in the optical and NIR bands were measured in fixed apertures of $3^{\prime \prime}$ diameter, on point-spread function (PSF) matched images (FWHM of 1".5). Monte Carlo simulations (Capak et al. 2007) have been used to correct for the flux potentially missed within the apertures. The 3".8 aperture fluxes given in the COSMOSIRAC catalog sources were also converted to total fluxes by using conversion factors taken from Surace et al. (2005). 
To make sure that confusion is not an issue in the IRAC and MIPS bands, we have visually inspected all IRAC and MIPS matches. In the IRAC bands, 82/89 objects have secure counterparts, and for them no obvious case of blending has been found when looking into $3^{\prime \prime}$ diameter circles centered on the source of the optical photometry: only one IRAC source is found in all objects, and the contaminating flux is usually lower than $1 \%$. For MIPS sources, the situation is slightly different. There we found four cases where the MIPS source could indeed be a blend of two IRAC sources lying within the MIPS error circle. We have thus decided to remove the MIPS photometric point for these objects. In any case, we have verified that our results are not changed, neither quantitatively nor qualitatively if we recalculate the stellar masses increasing the error uncertainty on all the $24 \mu \mathrm{m}$ points by $\pm 50 \%$.

In summary, apart from the 7 cases with ambiguous identification of the IRAC counterparts and the 4 confused MIPS sources, for all remaining 78 objects in our final sample, we have used 14 different bands that encompass optical to MIR wavelengths: 6 SUBARU bands $(B, V, g, r, i, z) ; U, J$, and $K$ bands from CFHT +4 Spitzer/IRAC bands $+24 \mu \mathrm{m}$ from Spitzer/MIPS. This allows us to sample a wide wavelength interval, ranging from $\sim 3800 \AA\left(\mathrm{U}_{\mathrm{CFHT}}\right)$ to $24 \mu \mathrm{m}$. All errors quoted are Poissonian.

\section{DISENTANGLING THE AGN AND HOST GALAXY EMISSION WITH SED FITTING}

One of the crucial goals of our study is to use the unprecedented multi-wavelength coverage of the COSMOS field to robustly derive host-galaxy properties through detailed model fitting of the total SED of the BLAGN in our sample. Salvato et al. (2009) have demonstrated how the COSMOS data allow the determination of reliable photometric redshifts by using composite AGN+galaxy templates to fit the multi-band photometry of all XMM-COSMOS sources (including both obscured and un-obscured AGNs). Here we apply a similar technique to our sample of BLAGNs (with known spectroscopic redshift) to try to unveil the physical properties of the galaxy component. We fit the observed SED with a relatively large grid of models made from a combination of AGN and host-galaxy templates. For the AGN component, we adopt the Richards et al. (2006) mean QSO SED (but see Section 6.3 for a discussion of possible alternative choices), as derived from the study of 259 IR-selected quasars with both Sloan Digital Sky Survey and Spitzer photometry. We allow for extinction of the nuclear AGN light applying an SMC-like dust-reddening law (Prevot et al. 1984) of the form: $A_{\lambda} / E(B-V)=1.39 \lambda_{\mu \mathrm{m}}^{-1.2}$ for $E(B-V)$ (reddening factor) values in the range $0 \leqslant E(B-V) \leqslant 0.3$. For the host-galaxy component, we adopt two different sets of templates:

1. First of all, we use the library of (observed) galaxy templates produced by the SWIRE survey (see Polletta et al. 2007, hereafter P07). From the entire library available of 25 templates, we excluded the AGN and composites (starburst+AGN), thus retaining only 14 templates (3 ellipticals, 7 spirals, and 4 starburst). Such a fitting algorithm has four free parameters: two normalizations for the AGN and galaxy templates, respectively, and two corresponding reddening factors.

2. We also created our own library of synthetic spectra using the well known models of stellar population synthesis of Bruzual \& Charlot (2003, hereafter BC03). Similarly to previous studies of the galaxy population in COSMOS (Ilbert et al. 2009; Bolzonella et al. 2009), we built 10 exponentially declining star formation histories (SFHs) $\mathrm{SFR} \propto e^{-t_{\text {age }} / \tau}$ with e-folding times, $\tau$, ranging from 0.1 to $30 \mathrm{Gyr}$, plus a model with constant star formation. For each of these SFHs, we calculate the synthetic spectrum at different ages, $t_{\text {age }}$, ranging from $50 \mathrm{Myr}$ to $5 \mathrm{Gyr}$, subject only to the constraint that the age should be smaller than the age of the universe at the redshift of the source. Finally, we allow for dust extinction, modeled by means of Calzetti's law (Calzetti et al. 2000), with values in the range $0 \leqslant E(B-V) \leqslant 0.5$. Following Fontana et al. (2004) and Pozzetti et al. (2007), we impose the prior $E(B-V)<0.15$ if $t_{\text {age }} / \tau>4$ (a significant extinction is only allowed for galaxies with a high star formation rate (SFR)). We adopt a Salpeter (1955) initial mass function (IMF) to compute stellar masses. Different choices of IMF lead to systematic shifts in the estimated stellar masses for any given SED (with the maximum shift for a Chabrier IMF (Chabrier 2002) given by $M_{*, \text { Chabrier }} \approx M_{*, \text { Salpeter }} / 1.8$; see, e.g., Pozzetti et al. 2007; Ilbert et al. 2009). We will discuss the effect of the IMF on our results in Section 6. Such a fitting algorithm has six free parameters: two normalizations for the AGN and galaxy templates, respectively, two corresponding reddening factors, and the age and e-folding time of the exponentially declining SFH.

We examined the global SED of each object and we fit the observed fluxes using a combination of the AGN and galaxy emission using the templates extracted from our libraries. Nine examples of the SED fitting are shown in Figure 1 (a gallery of all 89 SEDs can be found at www.mpe.mpg.de/ am/ plot_sed_all_rev.pdf).

For each parameter of interest $\left(M_{K}, M_{*}\right)$, we compute the onedimensional $\chi^{2}$ distribution obtained marginalizing over all the other parameters (with flat priors). The normalized probability distributions of $M_{K}\left(P \propto \exp \left(-\chi_{\text {red }}^{2} / 2\right)\right)$ for a few objects are shown as insets in Figure 1. As a general rule, because we are fitting the data with the sum of two model components, the probability distributions are asymmetric, with sometimes large tails toward small values of these parameters, corresponding to the cases in which the fitting procedure does not require with high significance the galaxy component besides the AGN one. We determine the best-fit value of the parameter of interest as the value that minimizes the $\chi^{2}$. One sigma errors on the best-fit parameters are computed rescaling the observational uncertainties until the minimum reduced $\chi_{\text {red }}^{2}=1$ (for a number of degrees of freedom equal to 8 for 79 objects, 7 for four without MIPS, and 5 for the seven without clear IRAC counterparts), and then finding the range in the parameter values within which $\Delta \chi^{2} \leqslant 1$. We then assign just an upper limit to the host-galaxy rest-frame $K$-band magnitude in all those cases where, within the above-mentioned uncertainty (i.e., where $\Delta \chi^{2} \leqslant 1$ ), the rest-frame $K$-band luminosity can have values smaller than a fixed fraction $f_{\text {gal }}$ of the corresponding AGN luminosity in the same rest-frame band. By inspection of the best-fit SED, after a number of trials, we fix $f_{\text {gal }}=0.05$; simply put, if we find a significant probability that the galaxy component is smaller than $5 \%$ of the AGN one, we decide that only an upper limit to the galaxy luminosity can be assigned. These 5\% limit values are marked as vertical red lines in the insets of Figure 1. Increasing such a threshold to 0.1 , although almost doubling the number of objects with only upper limits on the host-galaxy $M_{K}$, hardly produce any significant change in the general results and in the global trends discussed in Section 5. In those cases, the value of the upper limit is taken as the (non-zero) value of 


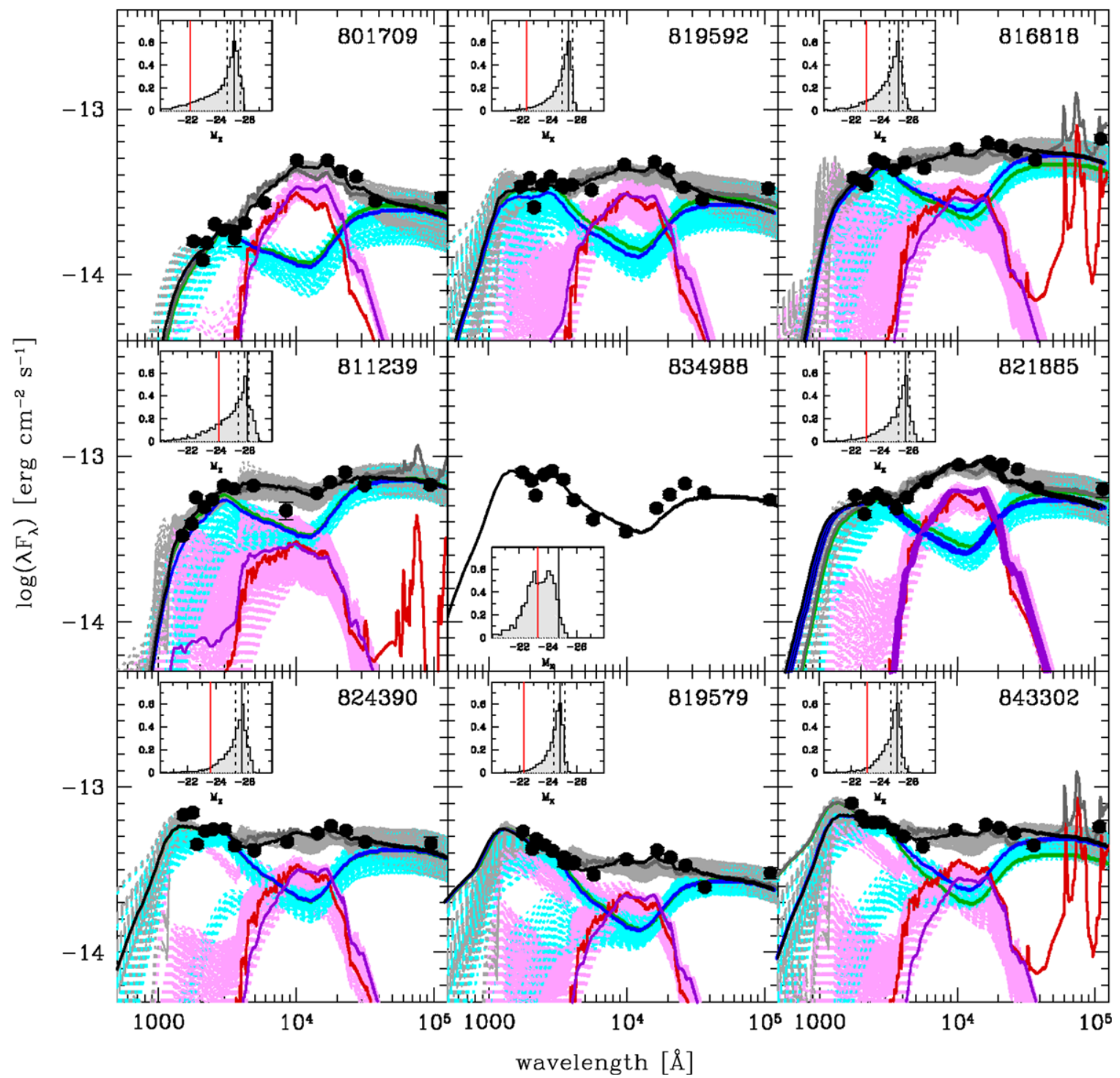

Figure 1. Examples of SED decompositions. Black circles are rest-frame fluxes corresponding to the 14 bands used to constrain the SED of each object. Purple and blue lines correspond respectively to the galaxy and the AGN template found as best-fit solution through the $\chi^{2}$ minimization for the BC03 template set (red and dark green for the P07 one), while the black line shows their sum (dark gray for P07 total). Pink and cyan dotted lines show the range of allowed SED from the BC03 template library within $1 \sigma$ of the best-fit $M_{K}$ measure, and light gray their sum. For one objects (VIMOS IDs 834988) our fitting procedure returns only an upper limit for the galaxy rest-frame $K$-band magnitude (overall 10/89); in this case we only plot the AGN spectral component. The inset in each panel shows the normalized probability distribution, $P=\exp \left(-\chi_{\text {red }}^{2} / 2\right)$, for the rest-frame $K$-band absolute magnitude of the host galaxy, with the solid vertical line marking the best-fit value and the dashed lines the $1 \sigma$ uncertainties. The red vertical line marks instead 5\% of the $K$-band magnitude of the AGN component. The full set of images of SED decomposition can be found at http://www.mpe.mpg.de/ am/plot_sed_all_rev.pdf

the parameter associated with the highest possible value of $M_{K}$ within the uncertainty. Finally, whenever we decide that only an upper limit can be meaningfully associated with the $K$-band luminosity of the host galaxy, we assign an upper limit to the object's total stellar mass adopting the median mass-to-light ratio of all other objects in the sample (this corresponds to adopting the following relation between the logarithm of the total stellar mass and $M_{K}$ for the upper limits in the sample: $\log M_{*}=-0.55-0.4\left(M_{K}-3.28\right)$, see Section 3.2). In total, for 10/89 objects we can provide only upper limits for the hostgalaxy SED component (and thus for $M_{K}$ and $M_{*}$ ).

\subsection{Rest-frame K-band Luminosities}

We are mainly interested here in determining the total mass of the host. It is generally believed that local scaling relations apply only when the bulge/spheroid component of the host galaxy is considered (Kormendy \& Gebhardt 2001; but see the recent works of Kim et al. 2008; Bennert et al. 2009, for a different view); however, reliable bulge-disk decomposition for our AGN hosts are problematic and will not be considered here; we will briefly discuss the implications of this issue later, in Section 6.1. In most of the objects of the sample the nuclear AGN emission dominates the emission in all optical bands, and the constraints on the host-galaxy emission are derived mostly in the wavelength range where the AGN SED has a minimum, around $1.2 \mu \mathrm{m}$, (Elvis et al. 1994; Richards et al. 2006). This is close to the rest-frame $K$ band, which is itself a good (i.e., such that the mass-to-light ratio in the $K$ band has a $1 \sigma$ scatter of about $0.1 \mathrm{dex}$ ) indicator of total mass (see, e.g., Madau et al. 1998; Bell et al. 2003). We thus proceed in two steps: we first try and constrain the rest-frame $K$ band magnitude of the AGN hosts, 
$M_{K}$, for a comparison with the local $M_{\mathrm{BH}}-M_{K}$ scaling relations (Marconi \& Hunt 2003; Graham 2007). Then, we proceed to derive the confidence interval on the measure of the total stellar mass.

As a first step, we verified that, given our choice of the AGN template, the derived $K$-band rest-frame magnitudes of the host galaxies are not sensitive to the particular set of galaxy SED templates used (P07 versus BC03). The average $1 \sigma$ errors in the estimated $M_{K}$ depend on the model SED used to fit the data, and are approximately 0.4 and 0.3 magnitudes for $\mathrm{BC} 03$ and $\mathrm{P} 07$, respectively. Keeping this in mind, we find a good agreement between these two methods: the difference in $M_{K}$ between the estimates obtained using the P07 templates and those obtained using the $\mathrm{BC} 03$ ones is strongly peaked at around zero, with small scatter (of the order of $0.3 \mathrm{dex}$ ), with the exception of $8 / 89$ outliers (i.e., objects with $\left|\Delta M_{K}\right|>0.7$, larger than the sum of typical $1 \sigma$ errors in $M_{K}$ ). The estimated $M_{K}$ (in Vega, ${ }^{27}$ for the fits with the $\mathrm{BC} 03$ templates) are given in Table 1, while the distribution of the estimated $M_{K}$ is shown in the upper panel of Figure 2. There, as a term of reference, we have also plotted the corresponding distribution of the rest-frame $K$-band magnitudes of the AGN components from our fits. Typically, AGNs are 1-2 magnitudes brighter than their hosts in the $K$ band; thus, the fraction of $K$-band light contributed by the host galaxy has a median of about $25 \%-30 \%$, with $90 \%$ of the object having this ratio smaller than 0.4 . The ratio $f_{\mathrm{gal}, \mathrm{K}} \equiv \frac{L_{\mathrm{gal}}}{L_{\mathrm{AGN}}}$ of the galaxy-to-AGN luminosity in the rest-frame $K$ band is also given in Table 1.

Encouraged by the robustness of the $M_{K}$ determination for the AGN hosts in our sample, we proceed to the discussion of the stellar mass estimates, and refer the reader to Section 6.3 for a further discussion of how a different choice of AGN SED template could modify our results.

\subsection{Host-galaxy Masses}

The fitting procedure with the $\mathrm{BC} 03$ templates allows us to estimate also the total stellar mass of the AGN hosts: each combination of SFH, $\tau$, and $t_{\text {age }}$ (see Section 3) is uniquely associated with a value of SFR and total stellar mass, computed taking into account the effect of stellar mass loss. The distribution of these stellar mass measurements is shown in the right panel of Figure 2. The estimated $\log M_{*}$ (for the fits with the $\mathrm{BC} 03$ templates) are also given in Table 1.

What kind of galaxies are these? As we will discuss in more detail later on, it is extremely difficult to extract reliable information on the star-forming properties of these objects, due to the dominant presence of the AGN emission. A more statistical comparison can be made by comparing the inferred masses with the overall galaxy mass function in the same redshift range as obtained by the S-COSMOS survey (duly shifted in mass to account for the difference in the average stellar mass between the Chabrier and Salpeter IMF; see Ilbert et al. 2009). We do not detect any galaxy with mass larger than $10^{11.6} M_{\odot}$, and most of our objects have stellar masses between $10^{10.5}$ and $10^{11.3} M_{\odot}$. According to the S-COSMOS mass functions (Ilbert et al. 2009), within this $M_{*}$ range, only less than $\sim 40 \%, 35 \%$, and $20 \%$ of all galaxies are "quiescent" (i.e., lie on the red sequence) for redshift ranges [1,1.2], [1.2, 1.5], and [1.5, 2.0], respectively.

\footnotetext{
${ }^{27}$ Since our magnitudes were all calibrated on the AB system, in order to ease the comparison with literature work, we use the following conversion between Vega and AB COSMOS $K$-band magnitudes: $M_{K \text {, Vega }}=M_{K, \mathrm{AB}}-1.84$.
}

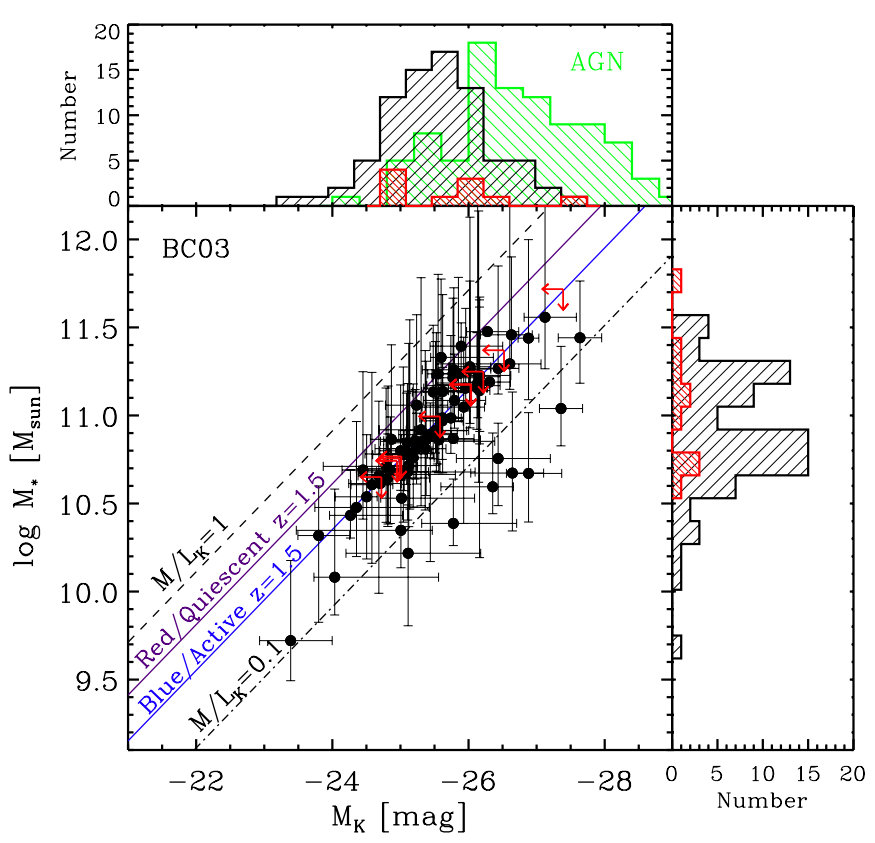

Figure 2. Estimated rest-frame $K$-band absolute magnitude vs. total stellar mass for the type-1 AGN hosts of our sample (large panel). Black solid circles are measures, red arrows upper limits. As a reference, lines of constant mass-to-light ratio $\left(M / L_{K}\right)$ equal to 1 and 0.1 are plotted as dashed and dot-dashed lines, respectively. The blue line is the average mass-to-light ratio for blue/actively star-forming galaxies at $z=1.5$ computed with the Arnouts et al. (2007) relation and shifted by -0.1 dex to make it consistent with the S-COSMOS results (Ilbert et al. 2009): $\log M / L_{K}=0.27 z-0.15$. The purple line is the average mass-to-light ratio for red/quiescent galaxies at $z=1.5$ computed with the Arnouts et al. (2007) relation, also shifted by -0.1 dex (Ilbert et al. 2009): $\log M / L_{K}=0.17 z-0.05$. The upper small panel shows the distribution of $M_{K}$ (black histogram: detections, red histogram: upper limits) together with the distribution of AGN $K$-band rest-frame magnitudes (green histogram). In the right small panel, the distribution of the measured stellar masses is displayed.

(A color version of this figure is available in the online journal.)

A similar conclusion about the average star-formation properties of the type-1 AGN hosts can be drawn by studying the mass-to-light ratio of the best-fitting models. Figure 2 shows the relation between rest-frame $K$-band luminosity and total stellar mass. Once again, we can compare these estimates of the $M / L_{K}$ ratio with those measured by Ilbert et al. (2009) in the COSMOS IR-selected galaxy sample (see Ilbert et al. 2009, appendix D). Taking into account the $\approx 0.24$ dex shift due to the different IMF choices, our mean $\log \left(M / L_{K}\right) \sim-0.55$ follows more closely the expectations for the so-called blue-cloud (star-forming) galaxies, but the nominal uncertainties remain large. ${ }^{28}$

As a further test of our method, we have compared the total stellar mass estimates with those derived by Jahnke et al. (2009) for a small sample (18 objects) of X-ray-selected AGNs in the COSMOS field for which simultaneous HST/ACS and $H S T /$ NICMOS observations allow us to derive mass-to-light ratios (and stellar masses) of the resolved hosts. We find that the two independent methods are in broad agreement with each other. 5/18 of them have also zCOSMOS spectra, and are part of the sample described here (see the empty stars in Figure 5). Their total stellar masses calculated with the two methods agree to better than $0.2 \mathrm{dex}$, with our estimates being

\footnotetext{
${ }^{28}$ We note here that the level of uncertainty of the SFR for our AGN hosts is such that we cannot clearly discriminate between what, in the galaxy formation jargon, is usually called "blue cloud" and "green valley" (see, e.g., Silverman et al. 2008).
} 
Table 1

Rest-frame $K$-band Magnitude and Total Stellar Masses of BLAGN Hosts in COSMOS

\begin{tabular}{|c|c|c|c|c|c|c|c|}
\hline VIMOS ID & R.A. (deg) & Decl. (deg) & $z$ & $M_{K}($ Vega $)$ & $f_{\mathrm{gal}, \mathrm{K}}{ }^{\mathrm{a}}$ & $\log M_{*}\left(M_{\odot}\right)$ & $\mathrm{ul}^{\mathrm{b}}$ \\
\hline 801709 & 149.985992 & 1.617284 & 1.126 & -25.24 & 0.847 & 11.06 & 0 \\
\hline 803695 & 150.596069 & 1.787450 & 1.246 & -24.81 & 0.281 & 10.70 & 0 \\
\hline 805949 & 150.050400 & 1.744427 & 1.149 & -25.74 & 1.459 & 10.99 & 0 \\
\hline 807560 & 149.687286 & 1.719174 & 1.349 & -24.27 & 0.077 & 10.43 & 0 \\
\hline 808150 & 149.545349 & 1.668093 & 2.096 & -26.88 & 0.446 & 11.44 & 0 \\
\hline 810061 & 150.536713 & 1.849565 & 1.824 & -24.35 & 0.142 & 10.48 & 0 \\
\hline 811239 & 150.278976 & 1.959607 & 1.550 & -26.15 & 0.307 & 11.18 & 0 \\
\hline 811646 & 150.195053 & 1.793735 & 1.851 & -26.35 & 0.317 & 10.59 & 0 \\
\hline 811799 & 150.161789 & 1.877919 & 1.444 & -24.68 & 0.536 & 10.66 & 0 \\
\hline 811904 & 150.141220 & 1.819711 & 1.192 & -25.01 & 0.310 & 10.35 & 0 \\
\hline 811960 & 150.131744 & 1.799389 & 1.675 & -25.53 & 0.372 & 10.90 & 0 \\
\hline 813283 & 149.821960 & 1.838634 & 1.351 & -25.96 & 0.408 & 11.16 & 0 \\
\hline 813416 & 149.791794 & 1.872849 & 1.567 & -25.77 & 0.596 & 11.21 & 0 \\
\hline 813886 & 149.687500 & 1.812649 & 1.215 & -24.45 & 0.596 & 10.69 & 0 \\
\hline 814414 & 149.564636 & 1.823087 & 1.507 & -26.22 & 0.170 & 11.25 & 1 \\
\hline 816818 & 150.446045 & 2.043490 & 1.171 & -25.30 & 0.391 & 10.92 & 0 \\
\hline 817202 & 150.386765 & 1.966629 & 1.537 & -25.62 & 0.415 & 10.99 & 0 \\
\hline 817260 & 150.373642 & 2.112055 & 1.914 & -25.02 & 0.096 & 10.68 & 0 \\
\hline 817480 & 150.328293 & 2.124951 & 1.780 & -25.78 & 0.425 & 10.87 & 0 \\
\hline 818094 & 150.195587 & 2.004415 & 1.923 & -26.52 & 0.182 & 11.37 & 1 \\
\hline 819187 & 149.957733 & 2.003069 & 1.806 & -25.12 & 0.168 & 10.72 & 0 \\
\hline 819193 & 149.955856 & 2.028046 & 1.756 & -27.64 & 0.579 & 11.44 & 0 \\
\hline 819446 & 149.897934 & 2.093906 & 1.910 & -25.11 & 0.377 & 10.68 & 0 \\
\hline 819579 & 149.868561 & 1.992970 & 1.166 & -24.85 & 0.523 & 10.69 & 0 \\
\hline 819592 & 149.865585 & 2.003061 & 1.248 & -25.44 & 0.742 & 10.89 & 0 \\
\hline 819644 & 149.851959 & 1.998422 & 1.244 & -26.88 & 0.326 & 10.67 & 0 \\
\hline 819702 & 149.837067 & 2.008842 & 1.481 & -24.82 & 0.143 & 10.64 & 0 \\
\hline 820341 & 149.663605 & 2.085205 & 1.220 & -25.10 & 0.273 & 10.85 & 0 \\
\hline 820375 & 149.656326 & 2.051113 & 1.855 & -25.14 & 0.586 & 10.78 & 0 \\
\hline 820673 & 149.586609 & 2.037102 & 1.850 & -25.04 & 0.149 & 10.67 & 0 \\
\hline 820679 & 149.585220 & 2.051113 & 1.355 & -25.54 & 0.137 & 10.92 & 0 \\
\hline 821039 & 149.506729 & 2.074688 & 1.226 & -25.55 & 0.571 & 11.24 & 0 \\
\hline 821885 & 150.708008 & 2.292316 & 1.099 & -25.78 & 0.607 & 11.26 & 0 \\
\hline 822461 & 150.581863 & 2.287697 & 1.343 & -25.89 & 0.239 & 11.39 & 0 \\
\hline 822703 & 150.536163 & 2.273239 & 1.087 & -25.61 & 1.854 & 10.97 & 0 \\
\hline 823199 & 150.451859 & 2.144812 & 1.298 & -25.00 & 0.206 & 10.80 & 0 \\
\hline 823714 & 150.345932 & 2.147529 & 1.258 & -26.16 & 0.841 & 11.22 & 0 \\
\hline 824176 & 150.236267 & 2.289114 & 2.078 & -25.11 & 0.147 & 10.22 & 0 \\
\hline 824306 & 150.214676 & 2.204261 & 1.841 & -24.03 & 0.068 & 10.08 & 0 \\
\hline 824390 & 150.199768 & 2.190844 & 1.510 & -25.79 & 0.381 & 11.09 & 0 \\
\hline 824396 & 150.198990 & 2.132499 & 2.160 & -27.12 & 0.223 & 11.56 & 0 \\
\hline 824572 & 150.158371 & 2.139555 & 1.828 & -26.61 & 0.207 & 11.29 & 0 \\
\hline 825363 & 150.004471 & 2.237096 & 1.407 & -25.77 & 0.317 & 11.24 & 0 \\
\hline 825899 & 149.895416 & 2.239492 & 1.742 & -25.52 & 0.179 & 10.91 & 0 \\
\hline 825906 & 149.894852 & 2.174454 & 1.323 & -25.48 & 0.991 & 11.13 & 0 \\
\hline 827274 & 149.624283 & 2.180656 & 1.185 & -25.93 & 0.478 & 11.05 & 0 \\
\hline 829667 & 150.499130 & 2.444901 & 2.025 & -27.36 & 0.560 & 11.04 & 0 \\
\hline 829682 & 150.495667 & 2.412547 & 1.370 & -26.13 & 0.818 & 11.18 & 0 \\
\hline 830510 & 150.347702 & 2.390998 & 1.848 & -26.02 & 0.379 & 11.28 & 0 \\
\hline 831077 & 150.231812 & 2.363971 & 1.936 & -25.02 & 0.093 & 10.53 & 0 \\
\hline 832354 & 149.993912 & 2.301415 & 1.789 & -25.56 & 0.130 & 10.86 & 0 \\
\hline 832715 & 149.919785 & 2.327419 & 1.454 & -26.04 & 0.136 & 11.18 & 1 \\
\hline 832923 & 149.881012 & 2.450839 & 1.315 & -26.16 & 0.957 & 11.14 & 0 \\
\hline 832961 & 149.871841 & 2.342855 & 1.735 & -25.19 & 0.487 & 10.76 & 0 \\
\hline 832963 & 149.870712 & 2.417283 & 1.528 & -25.06 & 0.353 & 10.78 & 0 \\
\hline 833273 & 149.812943 & 2.345459 & 1.800 & -26.64 & 0.464 & 10.67 & 0 \\
\hline 833541 & 149.763458 & 2.334125 & 1.131 & -25.24 & 0.431 & 10.81 & 0 \\
\hline 833712 & 149.730255 & 2.453799 & 1.101 & -24.58 & 1.592 & 10.61 & 0 \\
\hline 833817 & 149.705872 & 2.419752 & 1.108 & -26.16 & 0.525 & 11.17 & 0 \\
\hline 834079 & 149.660278 & 2.410915 & 1.161 & -26.28 & 0.768 & 11.48 & 0 \\
\hline 834383 & 149.602066 & 2.392675 & 1.849 & -27.39 & 0.215 & 11.72 & 1 \\
\hline 834988 & 149.462845 & 2.356840 & 1.185 & -24.72 & 0.186 & 10.65 & 1 \\
\hline 835006 & 149.459167 & 2.430080 & 1.242 & -23.80 & 0.339 & 10.32 & 0 \\
\hline 835631 & 150.715118 & 2.484831 & 1.996 & -26.44 & 0.117 & 10.76 & 0 \\
\hline 835840 & 150.668900 & 2.516766 & 1.573 & -23.39 & 0.088 & 9.72 & 0 \\
\hline 836198 & 150.597351 & 2.617924 & 1.447 & -26.12 & 1.295 & 11.16 & 0 \\
\hline
\end{tabular}


Table 1

(Continued)

\begin{tabular}{|c|c|c|c|c|c|c|c|}
\hline VIMOS ID & R.A. (deg) & Decl. (deg) & $z$ & $M_{K}(\mathrm{Vega})$ & $f_{\mathrm{gal}, \mathrm{K}}^{\mathrm{a}}$ & $\log M_{*}\left(M_{\odot}\right)$ & $\mathrm{ul}^{\mathrm{b}}$ \\
\hline 836355 & 150.572617 & 2.499909 & 1.102 & -24.70 & 0.190 & 10.61 & 0 \\
\hline 837652 & 150.334427 & 2.561485 & 1.832 & -26.31 & 0.234 & 11.19 & 0 \\
\hline 837827 & 150.305588 & 2.602232 & 1.342 & -24.98 & 0.188 & 10.76 & 1 \\
\hline 837858 & 150.299744 & 2.506903 & 1.506 & -25.01 & 0.222 & 10.77 & 1 \\
\hline 838223 & 150.230820 & 2.578165 & 1.401 & -25.58 & 0.132 & 10.99 & 1 \\
\hline 838610 & 150.163803 & 2.597661 & 1.589 & -25.09 & 0.367 & 10.78 & 0 \\
\hline 839188 & 150.058716 & 2.477386 & 1.256 & -24.95 & 0.095 & 10.74 & 1 \\
\hline 839751 & 149.955612 & 2.502021 & 1.458 & -25.85 & 0.583 & 11.24 & 0 \\
\hline 841635 & 149.575638 & 2.575658 & 1.171 & -24.50 & 0.590 & 10.54 & 0 \\
\hline 843302 & 150.635406 & 2.649920 & 1.223 & -25.22 & 0.343 & 10.86 & 0 \\
\hline 843685 & 150.554871 & 2.641009 & 1.144 & -25.36 & 0.503 & 10.81 & 0 \\
\hline 844213 & 150.456650 & 2.648144 & 2.050 & -26.43 & 0.279 & 11.27 & 0 \\
\hline 845220 & 150.251328 & 2.737164 & 2.162 & -25.32 & 0.104 & 10.85 & 0 \\
\hline 845272 & 150.240799 & 2.659021 & 1.410 & -24.87 & 0.449 & 10.86 & 0 \\
\hline 845728 & 150.147079 & 2.717479 & 1.177 & -25.62 & 1.152 & 11.14 & 0 \\
\hline 845970 & 150.104462 & 2.691239 & 1.882 & -26.03 & 0.272 & 11.17 & 1 \\
\hline 846335 & 150.042480 & 2.629174 & 1.569 & -26.63 & 0.251 & 11.46 & 0 \\
\hline 847623 & 149.774170 & 2.674153 & 1.108 & -26.02 & 1.851 & 11.26 & 0 \\
\hline 848220 & 149.621902 & 2.738307 & 1.889 & -25.78 & 0.088 & 10.39 & 0 \\
\hline 851007 & 150.158981 & 2.825123 & 1.856 & -26.16 & 0.332 & 11.23 & 0 \\
\hline 900028 & 150.084366 & 2.290529 & 1.112 & -24.79 & 0.595 & 10.71 & 0 \\
\hline 900066 & 149.551071 & 2.316902 & 1.432 & -25.60 & 0.462 & 11.33 & 0 \\
\hline 950021 & 149.574463 & 2.085072 & 1.623 & -25.38 & 0.452 & 10.86 & 0 \\
\hline
\end{tabular}

Notes.

${ }^{a}$ Ratio of galaxy to AGN luminosity in the rest-frame $K$ band, as derived from the SED decomposition technique.

b Upper limit flag: $1=$ upper limit

consistently on the lower side. We discuss this issue further in Section 7.1.

\section{VIRIAL BLACK HOLE MASS MEASUREMENTS}

Due to the uncertainties in the actual geometry of the broadline region (BLR) of type-1 AGN (see, e.g., McLure \& Dunlop 2001), and/or to the different choice of absolute calibration of the black hole masses of local AGN studied with reverberation mapping, there exists a large number of different formulae in the literature that relate black hole mass, $\mathrm{Mg}$ II line width and continuum luminosity (see, e.g., McGill et al. 2008, and references therein). They can all be expressed in the form:

$$
\log \frac{M_{\mathrm{BH}}}{M_{\odot}}=A+\log \left(\mathrm{FWHM}_{1000}^{2}\left(\lambda L_{3000,44}\right)^{\beta}\right)
$$

where FWHM $_{1000}$ is the FWHM of the line in units of $1000 \mathrm{~km} \mathrm{~s}^{-1}$, and $\lambda L_{3000,44}$ is the continuum luminosity at $3000 \AA$ in units of $10^{44} \mathrm{erg} \mathrm{s}^{-1}$. For Mg II lines, McLure \& Dunlop (2004) proposed $A=6.51$ and $\beta=0.62$, based on a fit to the radius-luminosity relation for AGNs with reverberation mapping and total luminosity $\lambda L_{\lambda}>10^{44} \mathrm{erg} \mathrm{s}^{-1}$, while M. Vestergaard et al. (2009; in preparation; see also Trump et al. 2009) propose a different scaling $(A=6.86, \beta=0.5)$. Each of these relations carries a significant scatter of about 0.3 dex (McGill et al. 2008). The exponent $\beta$ is related to the empirical calibration of the radius-luminosity relation (Kaspi et al. 2000). A recent re-analysis of reverberation mapping observations, fully accounting for host-galaxy light contamination (Bentz et al. 2009), points toward a value of $0.45 \lesssim \beta \lesssim 0.59$, consistent with the simple expectations from photoionization models of the $\operatorname{BLR}(\beta=0.5)$. Here we will adopt the relation derived by McGill et al. (2008), with $A=6.77$ and $\beta=0.47$. This was derived by cross-calibrating a number of different estimators applied to BLAGNs in a redshift range where more than one broad emission line can be observed simultaneously in optical spectra. Additional systematic errors in the derived black hole masses introduced by the use of any of these relations will be further discussed in Section 4.3 (see also the discussion in Treu et al. 2007).

We do not take into account possible effects due to radiation pressure on the BLR, that could lead to systematic underestimate of the black hole mass for the objects with the higher Eddington ratio. Such an effect has been estimated empirically by Marconi et al. (2008) for the $\mathrm{H} \beta$ broad emission in a local AGN sample. No calibration is currently available for $\mathrm{Mg}$ II emission lines. Moreover, the size of the possible radiation pressure correction is expected to scale linearly with the inverse of the Eddington ratio, and should not be too large for the moderate-luminosity AGN in our sample (see Section 4.3 below). We thus made the conservative choice not to include any radiation pressure correction to the virial relation (1). As we will discuss at length in the following, this corresponds to minimize the amount of possible evolution detected in the average $M_{\mathrm{BH}} / M_{*}$ ratio as a function of redshift.

In the following subsections, we will describe the method used to measure both line width and continuum luminosity needed to apply Equation (1). The measured $F W H M_{1000}, L_{3000}$, together with their $1 \sigma$ errors, are given in Table 2.

\subsection{FWHM Measurement}

Type-1 (un-absorbed) AGN spectra in the wavelength region of interest are usually characterized by a power-law continuum, of the form $f_{\lambda} \propto \lambda^{\alpha}$, broad-line emission from Mg II plus a 
No. 1,2010

Table 2

Mg II Broad Emission Line FWHM, Continuum Luminosity, and Black Hole Mass for zCOSMOS Type-1 AGN

\begin{tabular}{|c|c|c|c|c|c|c|c|}
\hline VIMOS ID & $m_{i}^{\mathrm{a}}$ & $\mathrm{FWHM}_{1000}{ }^{\mathrm{b}}$ & $\Delta \mathrm{FWHM}$ & $L_{3000,40}{ }^{\mathrm{c}}$ & $\Delta L_{3000,40}$ & $\log M_{\mathrm{BH}}\left[M_{\odot}\right]^{\mathrm{d}}$ & Radio Flag ${ }^{\mathrm{e}}$ \\
\hline 801709 & 22.41 & $9.23^{\mathrm{f}}$ & 0.19 & 2.97 & 0.15 & 8.67 & RQ \\
\hline 803695 & 21.39 & 4.98 & 0.06 & 8.34 & 0.20 & 8.35 & RQ \\
\hline 805949 & 21.74 & 2.52 & 0.15 & 5.39 & 0.21 & 7.67 & RL \\
\hline 807560 & 20.36 & 7.82 & 0.21 & 37.48 & 0.48 & 9.05 & RQ \\
\hline 808150 & 20.20 & 3.45 & 0.11 & 85.33 & 5.14 & 8.51 & RQ \\
\hline 810061 & 21.71 & 4.69 & 0.41 & 18.64 & 1.10 & 8.46 & RQ \\
\hline 811239 & 20.83 & 2.63 & 0.05 & 33.89 & 0.92 & 8.08 & RQ \\
\hline 811646 & 21.99 & 5.48 & 0.41 & 15.78 & 1.51 & 8.56 & $\mathrm{RL}$ \\
\hline 811799 & 22.41 & 3.80 & 0.29 & 9.21 & 0.37 & 8.13 & RQ \\
\hline 811904 & 22.22 & 3.74 & 0.56 & 2.89 & 0.18 & 7.88 & RQ \\
\hline 811960 & 20.90 & 5.46 & 0.81 & 33.60 & 0.97 & 8.71 & RQ \\
\hline 813283 & 20.88 & 4.97 & 0.21 & 21.60 & 0.30 & 8.54 & RQ \\
\hline 813416 & 22.42 & 3.63 & 0.25 & 5.98 & 0.56 & 8.01 & RQ \\
\hline 813886 & 22.42 & 6.49 & 0.28 & 3.18 & 0.21 & 8.38 & RQ \\
\hline 814414 & 20.34 & 2.70 & 0.12 & 26.15 & 0.39 & 8.05 & RQ \\
\hline 816818 & 21.14 & 5.46 & 0.07 & 11.64 & 0.27 & 8.50 & RQ \\
\hline 817202 & 21.27 & 3.80 & 0.10 & 18.89 & 0.54 & 8.28 & RQ \\
\hline 817260 & 20.95 & 6.77 & 0.09 & 45.04 & 1.80 & 8.96 & RQ \\
\hline 817480 & 22.30 & 10.46 & 0.26 & 11.40 & 1.59 & 9.06 & RQ \\
\hline 818094 & 20.13 & 4.42 & 0.17 & 87.15 & 2.41 & 8.72 & RL \\
\hline 819187 & 21.03 & 5.29 & 0.06 & 33.82 & 1.63 & 8.69 & RQ \\
\hline 819193 & 19.00 & 4.46 & 0.21 & 221.60 & 2.12 & 8.92 & RQ \\
\hline 819446 & 21.73 & 3.35 & 0.15 & 14.75 & 1.53 & 8.12 & RQ \\
\hline 819579 & 21.69 & 3.95 & 0.14 & 7.83 & 0.27 & 8.13 & RQ \\
\hline 819592 & 21.90 & 3.82 & 0.11 & 6.55 & 0.21 & 8.07 & RQ \\
\hline 819644 & 18.53 & 3.65 & 0.45 & 139.86 & 6.38 & 8.65 & RQ \\
\hline 819702 & 20.93 & 3.56 & 0.16 & 24.83 & 0.63 & 8.28 & RQ \\
\hline 820341 & 20.62 & 4.69 & 0.58 & 22.64 & 0.31 & 8.50 & RQ \\
\hline 820375 & 22.45 & 5.02 & 0.16 & 8.07 & 0.77 & 8.35 & RQ \\
\hline 820673 & 21.06 & 4.97 & 0.07 & 25.19 & 1.34 & 8.57 & RQ \\
\hline 820679 & 19.82 & 5.39 & 0.07 & 63.30 & 0.65 & 8.83 & RQ \\
\hline 821039 & 22.01 & 5.97 & 0.43 & 4.79 & 0.20 & 8.39 & RQ \\
\hline 821885 & 20.99 & 5.48 & 0.19 & 12.85 & 0.22 & 8.52 & RQ \\
\hline 822461 & 21.29 & 7.93 & 0.52 & 14.01 & 0.32 & 8.86 & RQ \\
\hline 822703 & 21.58 & 5.88 & 0.31 & 6.60 & 0.22 & 8.45 & RQ \\
\hline 823199 & 20.61 & 3.94 & 0.13 & 26.23 & 0.40 & 8.38 & RQ \\
\hline 823714 & 20.95 & 2.68 & 0.21 & 19.24 & 0.34 & 7.98 & $\mathrm{RL}$ \\
\hline 824176 & 21.48 & 6.19 & 0.19 & 25.54 & 2.31 & 8.77 & RL \\
\hline 824306 & 20.99 & 2.68 & 0.10 & 27.69 & 1.14 & 8.05 & RQ \\
\hline 824390 & 21.12 & 4.90 & 0.27 & 19.09 & 0.65 & 8.50 & RQ \\
\hline 824396 & 19.49 & 3.62 & 0.19 & 108.25 & 4.59 & 8.59 & RQ \\
\hline 824572 & 20.45 & 4.27 & 0.47 & 52.42 & 1.44 & 8.59 & $\mathrm{RL}$ \\
\hline 825363 & 21.70 & 4.54 & 0.23 & 10.70 & 0.36 & 8.32 & RQ \\
\hline 825899 & 20.85 & 3.50 & 0.92 & 32.38 & 1.96 & 8.32 & RQ \\
\hline 825906 & 22.05 & 6.09 & 0.10 & 6.52 & 0.24 & 8.47 & RQ \\
\hline 827274 & 20.82 & 2.89 & 0.05 & 17.00 & 0.37 & 8.02 & RQ \\
\hline 829667 & 19.29 & 5.60 & 0.27 & 218.38 & 3.40 & 9.12 & RQ \\
\hline 829682 & 21.60 & 4.97 & 0.04 & 12.63 & 0.21 & 8.43 & RQ \\
\hline 830510 & 22.17 & 6.76 & 0.23 & 17.24 & 0.85 & 8.76 & RQ \\
\hline 831077 & 20.58 & 4.13 & 0.11 & 55.50 & 3.90 & 8.57 & RQ \\
\hline 832354 & 20.12 & 4.45 & 0.09 & 67.77 & 1.49 & 8.68 & RQ \\
\hline 832715 & 20.15 & 7.90 & 0.14 & 50.76 & 0.81 & 9.12 & $\mathrm{RL}$ \\
\hline 832923 & 21.29 & 3.59 & 0.26 & 15.58 & 0.35 & 8.19 & RL \\
\hline 832961 & 21.86 & 3.33 & 0.24 & 12.29 & 0.71 & 8.08 & RQ \\
\hline 832963 & 22.40 & 4.93 & 1.25 & 6.90 & 0.55 & 8.30 & RQ \\
\hline 833273 & 21.94 & 5.23 & 0.38 & 14.22 & 1.83 & 8.50 & RQ \\
\hline 833541 & 21.11 & 4.99 & 0.30 & 10.49 & 0.34 & 8.40 & RQ \\
\hline 833712 & 22.03 & 4.76 & 0.22 & 2.43 & 0.44 & 8.06 & RQ \\
\hline 833817 & 20.57 & 5.56 & 0.39 & 27.18 & 0.45 & 8.69 & RQ \\
\hline 834079 & 21.41 & 11.18 & 0.53 & 7.70 & 0.31 & 9.03 & RL \\
\hline 834383 & 19.86 & 5.32 & 0.12 & 111.41 & 1.68 & 8.94 & RQ \\
\hline 834988 & 20.57 & 5.53 & 0.21 & 18.92 & 0.39 & 8.61 & RQ \\
\hline 835006 & 22.20 & 1.93 & 0.10 & 5.64 & 0.28 & 7.45 & RQ \\
\hline 835631 & 19.54 & 2.31 & 0.10 & 144.30 & 2.77 & 8.26 & RQ \\
\hline 835840 & 22.29 & 3.19 & 0.33 & 6.17 & 0.75 & 7.90 & RQ \\
\hline 836198 & 21.76 & 3.92 & 0.12 & 10.76 & 0.35 & 8.19 & RQ \\
\hline
\end{tabular}


Table 2

(Continued)

\begin{tabular}{|c|c|c|c|c|c|c|c|}
\hline VIMOS ID & $m_{i}{ }^{\mathrm{a}}$ & FWHM $_{1000}{ }^{b}$ & $\Delta \mathrm{FWHM}$ & $L_{3000,40}{ }^{\mathrm{c}}$ & $\Delta L_{3000,40}$ & $\log M_{\mathrm{BH}}\left[M_{\odot}\right]^{\mathrm{d}}$ & Radio Flag $^{\mathrm{e}}$ \\
\hline 836355 & 20.68 & 5.10 & 0.14 & 17.32 & 0.22 & 8.52 & RQ \\
\hline 837652 & 20.08 & 5.90 & 0.11 & 76.87 & 1.86 & 8.95 & RQ \\
\hline 837827 & 22.06 & 3.82 & 0.10 & 6.02 & 0.28 & 8.05 & RQ \\
\hline 837858 & 21.02 & 3.54 & 0.09 & 24.02 & 1.40 & 8.27 & RQ \\
\hline 838223 & 20.05 & 4.31 & 0.06 & 54.27 & 0.92 & 8.61 & RL \\
\hline 838610 & 21.88 & 3.94 & 1.10 & 12.12 & 1.13 & 8.22 & RQ \\
\hline 839188 & 20.55 & 4.83 & 0.07 & 28.03 & 0.36 & 8.57 & RL \\
\hline 839751 & 22.11 & 9.04 & 0.74 & 5.90 & 0.76 & 8.80 & RQ \\
\hline 841635 & 21.72 & $5.80^{\mathrm{f}}$ & 0.41 & 7.01 & 0.23 & 8.45 & RQ \\
\hline 843302 & 21.15 & 3.82 & 0.21 & 16.72 & 0.27 & 8.26 & RQ \\
\hline 843685 & 21.53 & 5.55 & 0.11 & 6.48 & 0.22 & 8.39 & RQ \\
\hline 844213 & 20.47 & 4.76 & 0.36 & 62.15 & 2.02 & 8.72 & RQ \\
\hline 845220 & 20.85 & 3.92 & 0.20 & 25.43 & 3.22 & 8.37 & RQ \\
\hline 845272 & 22.49 & 3.79 & 0.26 & 5.59 & 0.42 & 8.03 & RQ \\
\hline 845728 & 22.19 & 6.19 & 0.16 & 3.43 & 0.29 & 8.36 & RQ \\
\hline 845970 & 21.08 & 3.79 & 0.20 & 35.96 & 2.41 & 8.41 & RQ \\
\hline 846335 & 20.21 & $4.30^{f}$ & 0.28 & 57.32 & 1.29 & 8.61 & RQ \\
\hline 847623 & 21.59 & 6.54 & 1.44 & 4.55 & 0.23 & 8.46 & RQ \\
\hline 848220 & 20.03 & 3.83 & 0.19 & 94.11 & 2.76 & 8.62 & RQ \\
\hline 851007 & 20.72 & 3.38 & 0.11 & 41.31 & 1.23 & 8.34 & RQ \\
\hline 900028 & 22.49 & 8.21 & 0.24 & 1.66 & 0.20 & 8.45 & RQ \\
\hline 900066 & 22.36 & 6.89 & 0.60 & 6.14 & 0.25 & 8.57 & RQ \\
\hline 950021 & 22.11 & 6.43 & 0.17 & 12.09 & 1.77 & 8.65 & RQ \\
\hline
\end{tabular}

Notes.

a Apparent $i$-band (AB) magnitude of the zCOSMOS spectroscopy target.

${ }^{\mathrm{b}} \mathrm{Mg}$ II emission line FWHM in units of $1000 \mathrm{~km} \mathrm{~s}^{-1}$.

c Monochromatic continuum AGN luminosity in units of $10^{40} \mathrm{erg} \mathrm{s}^{-1}$

${ }^{\mathrm{d}}$ Logarithm of the black hole mass, as computed with Equation (1), adopting the MG08 calibration (see text for details).

${ }^{\mathrm{e}} \mathrm{RQ}$ are objects detected in the VLA/COSMOS Survey at $1.4 \mathrm{GHz}$ (Bondi et al. 2008; Schinnerer et al. 2007).

${ }^{\mathrm{f}}$ Objects with clear narrow absorption features in the $\mathrm{Mg}$ II line.

complex of Fe II emission lines, also broadened at the typical velocities of the BLR (Boroson \& Green 1992; Vestergaard \& Wilkes 2001). Accurate subtraction of the broad Fe II features is thus an important step in the process of obtaining broad $\mathrm{Mg}$ II line widths. It is not always straightforward to keep track of the Fe II subtraction technique in previous studies high-redshift scaling relations. For example, Peng et al. (2006a) erroneously report that McLure \& Jarvis (2002) did not performed any Fe II subtraction, contrary to what stated in Section 3.2 of McLure \& Jarvis (2002). For the sake of completeness, we briefly discuss in Section 6.2 what would be the typical systematic effect of not removing an iron template from the spectra.

We adopt here the theoretical Fe II template calculated by Bruhweiler \& Verner (2008), calibrated by fitting the Seyfert 1 galaxy IZw1 spectrum (model Fe_d11-m20-20.5 available at http://iacs.cua.edu/people/verner/FeII). In order to apply the iron template to the spectra, the line width of the template must be matched to that of the AGN spectrum. We achieve this by means of an iterative procedure, as described below.

First of all, we convolved the original Fe II template with Gaussian functions of different widths, ranging from $v=$ $1000 \mathrm{~km} \mathrm{~s}^{-1}$ to $v=15,000 \mathrm{~km} \mathrm{~s}^{-1}$ in step of $250 \mathrm{~km} \mathrm{~s}^{-1}$ to produce a grid of broadened templates. From the original AGN spectrum we then derived: (1) a first rough estimation of the FWHM of the Mg II line using a single Gaussian fit with iraf-splot package and (2) the mean values of the continuum flux and the corresponding errors (derived from the noise spectra) in the following wavelength windows: [2660 $\mathrm{A}-2700 \AA$ ]; [2930 ̊-2970 ̊]; [2715
[2980 $\AA-3020 \AA] .{ }^{29}$ We assumed that in these wavelength ranges, the spectrum can be completely described by a combination of power law and Fe II emission smeared by a velocity width $v$ equal to the one derived from the $\mathrm{Mg}$ II emission line

$$
F(\lambda)=a \lambda^{\alpha}+b \times \operatorname{Fe~II~}(\lambda, v) .
$$

In order to find the best-fitting model, we then performed a chisquare minimization and we thus derive the three parameters $\alpha$, $a$, and $b$. Finally, we subtracted the best-fit power-law plus Fe II model to the original spectrum obtaining a new spectrum that contains only the Mg II emission line. This is done using the iraf-sarith package.

We then measured the FWHM of the line, modeled using one, two, or three Gaussians (absorption and/or emission) and we choose the best solution according to best reduced $\chi^{2}$ computed in the [2650 $\left.\AA-2950 \AA\right]$ range. The best-fit model for the emission line spectrum is thus given by $M_{\lambda}=$ $\sum_{i=0}^{N} a_{i} G_{\lambda}\left(\lambda_{\text {peak }, i}, \sigma_{i}\right)$, where $N$ is the number of Gaussian components chosen by the fit. For each Gaussian component $G_{\lambda}$, $a_{i}$ is the intensity (positive for emission, negative for absorption), $\lambda_{\text {peak }, i}$ the wavelength of the peak and $\sigma_{i}$ the width. Each of these quantities carries a statistical error provided by the splot-iraf package.

\footnotetext{
29 For a handful of objects (5) with a relatively small FWHM, a significant improvement in the fit is obtained by shifting the two central windows for continuum plus Fe II fitting to [2730 ̊-2770 ̊] ; [2830 ̊-2870 ̊], closer to the most prominent emission peaks of the Fe II complex.
} 

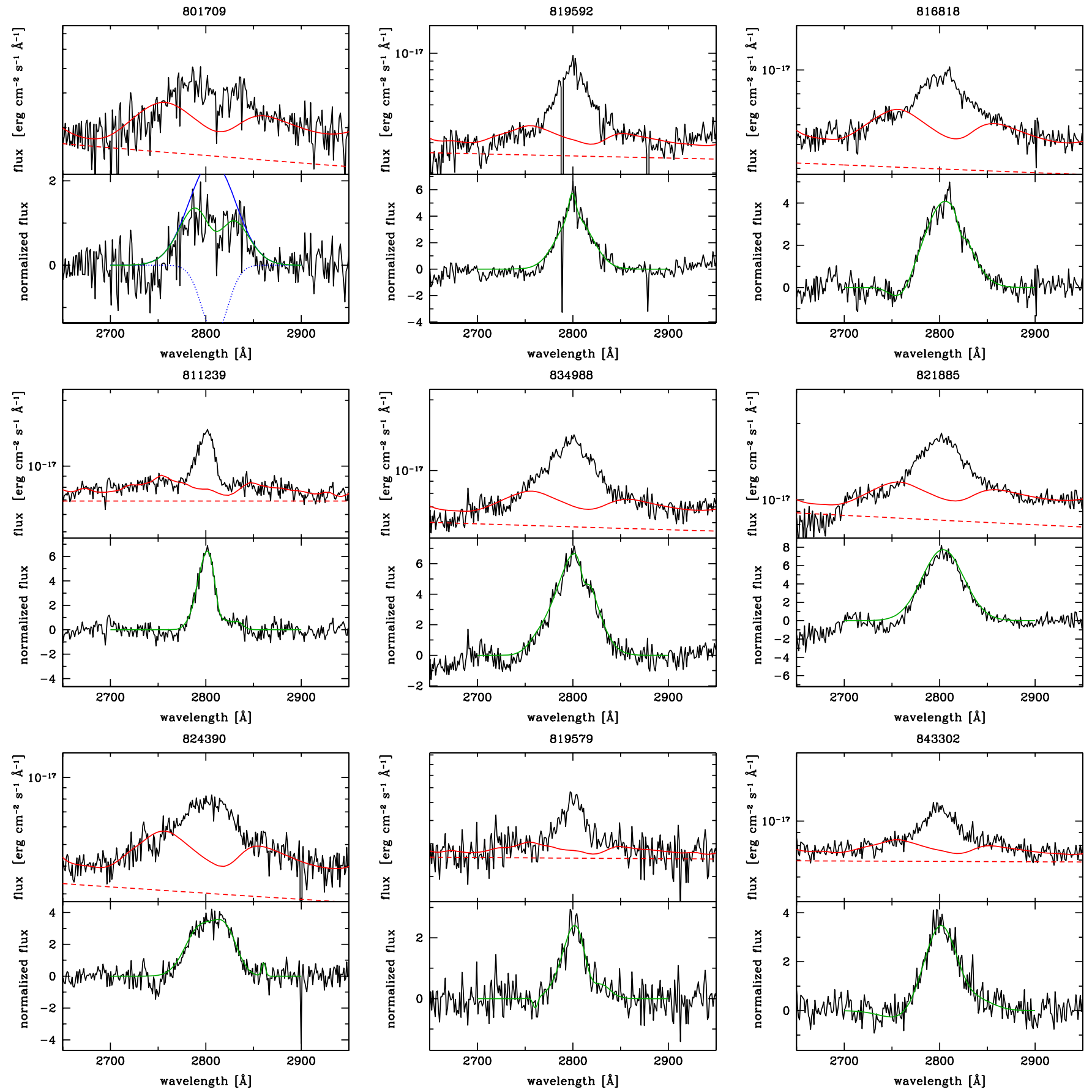

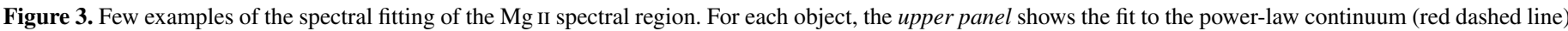

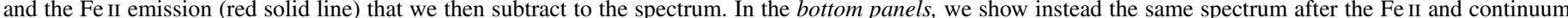

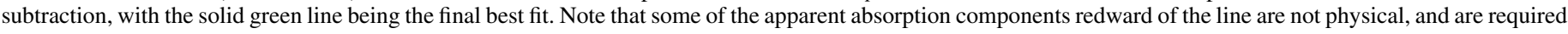

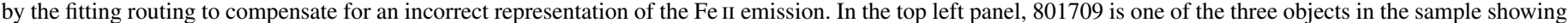

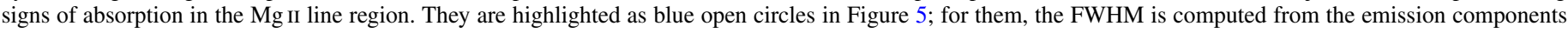
only.

(A color version of this figure is available in the online journal.)

We then compute the FWHM of the model emission line complex, and the error on the total FWHM, $\sigma_{\mathrm{FWHM}}$ is computed by propagating the errors on the single Gaussian components of the fit. Using the new FWHM determination for the line, we then iterated the whole procedure (we subtract the new broadened iron template chosen according to the FWHM measured from the original spectrum), until the fits converge and the final measure of the FWHM is stable. The final FWHM measurement is corrected for the finite spectrograph resolution assuming that $\mathrm{FWHM}_{\text {intrinsic }}^{2}=\mathrm{FWHM}_{\mathrm{oss}}^{2}-\frac{\lambda_{\text {eff }}^{2}}{R^{2}}$ where $R$ is the mean instrumental resolution that for the zCOSMOS spectra is $\simeq 580$. The best-fit decomposition of the Mg II region of the spectra is shown in Figure 3 for the same nine AGNs whose SED decomposition is shown in Figure 1. For three objects the fitting routine requires the presence of absorption in the $\mathrm{Mg}$ II line region (one is shown in the top left panel in Figure 3). They are 


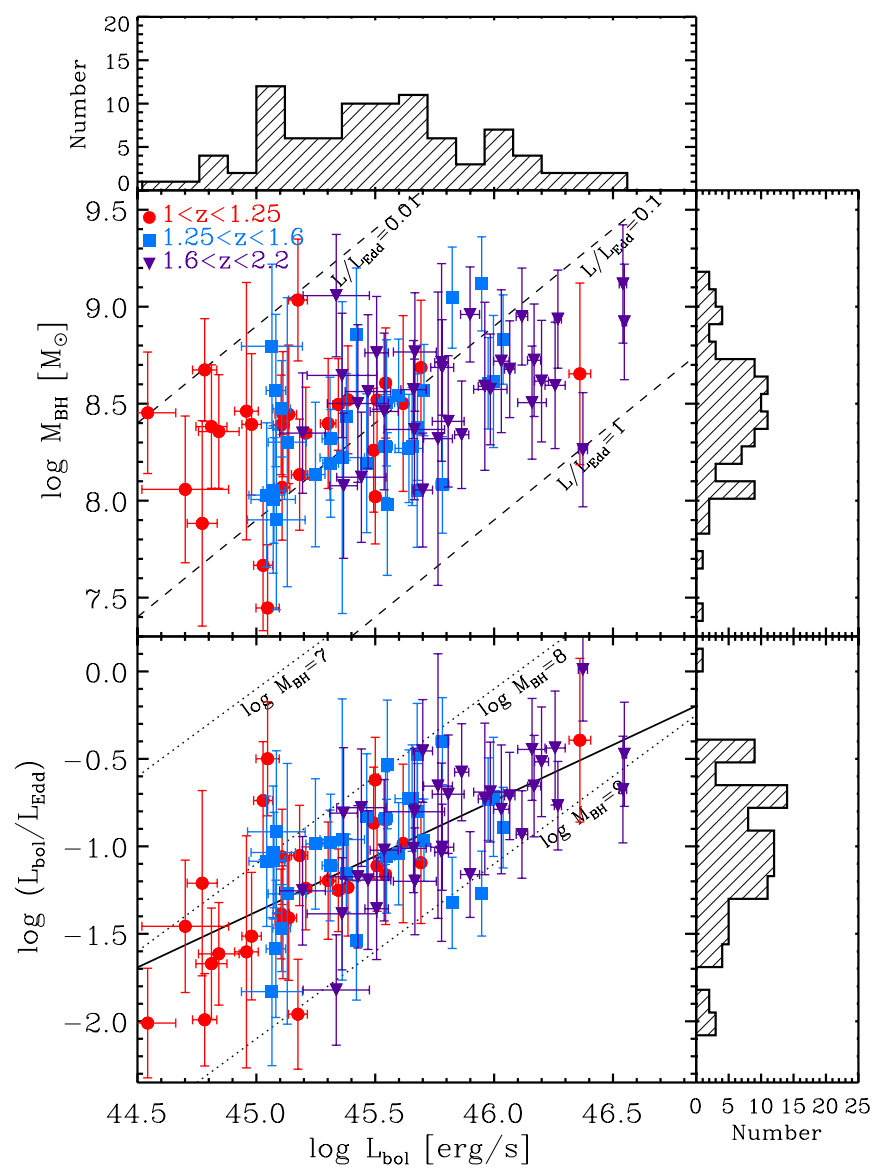

Figure 4. Large top panel: black hole mass vs. bolometric luminosity for the zCOSMOS BLAGNs in the redshift range $1<z<2.2$. Different colors and symbols correspond to different redshift ranges, while dashed lines mark the loci of constant Eddington ratios. Large bottom panel: Eddington ratio vs. bolometric luminosity for the same objects. Dotted lines mark the loci of constant BH mass, while the black solid line is the best-fit linear regression to the data points, given by $\log \left(L_{\text {bol }} / L_{\text {Edd }}\right)=-1.38+0.64 \log L_{\text {bol }, 45}$, where $L_{\text {bol }, 45}$ is the bolometric luminosity in units of $10^{45} \mathrm{erg} \mathrm{s}^{-1}$. The small panels in the top and right-hand side display the distributions of bolometric luminosity, black hole mass, and Eddington ratio, respectively.

(A color version of this figure is available in the online journal.)

marked as blue open circles in Figure 5. Some residual errors due to oversubtraction of the iron template redward of the $\mathrm{Mg}$ II emission are apparent in some cases. It is clear that a single Fe II template may not be adequate to fully describe this complex emission in all objects. A detailed study of the properties of the Fe II emission requires higher signal-to-noise spectra, and is beyond the scope of the present paper.

\section{2. $3000 \AA$ And Bolometric Luminosity}

From the best-fitting power-law continuum we derive an estimate of the AGN luminosity at $3000 \AA$. Our points have been corrected for aperture effects normalizing the $i$-band fluxes measured in the VIMOS spectra with the observed ACS $i$-band photometry. Monochromatic continuum luminosities at $3000 \AA$ were then calculated from the average best-fit continuum flux rescaled in the 2980-3020 $\AA$ rest frame. The error on the continuum luminosity is obtained from the average of the noise spectrum in the same wavelength range.

From the measured total $\lambda L_{3000}$ AGN luminosity we derive the bolometric one using the luminosity-dependent bolometric correction factor $f_{\text {bol }}$ of Hopkins et al. (2007b). The distribution of bolometric luminosities for the type-1 AGNs in our sample is shown in the upper panel of Figure 4. Assuming a standard radiative efficiency of $\epsilon_{\mathrm{rad}}=0.1$, the median accretion rate onto the black holes, $\dot{M}=L_{\mathrm{bol}} / \epsilon_{\mathrm{rad}} c^{2}$, is of the order of $0.4 M_{\odot} / \mathrm{yr}$.

\subsection{Black Hole Masses and Eddington Ratio Distribution}

We have tested the impact of the choice of single-epoch virial formula for the black hole mass uncertainty. The typical spread in $\log M_{\mathrm{BH}}$ among the three different estimators discussed in Section 4 is of the order of 0.2 dex, similar to the observed scatter in the virial relations themselves (Vestergaard \& Peterson 2006; McGill et al. 2008). In the following, we fix the systematic uncertainty in the $\log M_{\mathrm{BH}}$ determination to $0.2 \mathrm{dex}$, and for our statistical analysis of the scaling relation evolution, we assign to each black hole mass measurement an error given by the sum of the statistical and systematic uncertainties.

Figure 4 shows the estimated black hole masses versus the bolometric luminosities for all the objects in our sample (upper panel) as well as their location in the Luminosity-Eddington ratio $\left(\lambda \equiv L_{\text {bol }} / L_{\text {Edd }}\right.$, where $L_{\text {Edd }}=1.3 \times 10^{38} M_{\mathrm{BH}} / M_{\odot}$ is the Eddington luminosity) plane in the lower panel. As typical for optically selected samples of type-1 AGNs/QSOs (Kollmeier et al. 2006; Gavignaud et al. 2008; Trump et al. 2009, and references therein), the distribution of Eddington ratios is quite narrowly distributed, with a median of $\approx 0.1$.

Analogously to what found in BLAGN samples selected in similar redshift ranges and at comparable depths (VVDS; Gavignaud et al. 2008), we also observe a trend of increasing Eddington ratio with increasing bolometric luminosity of the AGN (see also Netzer et al. 2007, for a sample of higher-redshift QSOs). Fitting a straight line we find $\log \lambda \propto 0.64 \log L_{\text {bol }}$, but the slope of such a relation depends critically on the exponent $\beta$ in the adopted virial relation (1). As already pointed out in Gavignaud et al. (2008), if we chose the McLure \& Dunlop (2004) formula (with $\beta=0.62$ ), we would obtain a shallower slope: $\log \lambda \propto 0.47 \log L_{\text {bol }}$. Selection effects could certainly be playing a role in determining the distribution of sources in the $\lambda-L_{\text {bol }}$ plane (for example, objects in the lower right corner, i.e., massive black holes at low accretion rates, probe the massive end of the SMBH mass function, which is rapidly declining in this redshift range, see, e.g., Merloni \& Heinz 2008). The detailed distribution of AGN lifetimes as a function of luminosity can also be responsible for the observed trends, as suggested by some numerical models for QSO light curves (Hopkins \& Hernquist 2009). For further discussion of possible selection and/or systematics effects, we refer the reader to the work of Trump et al. (2009), who have studied in greater detail the larger sample of the XMM-COSMOS AGNs with IMACS spectroscopy.

\section{SCALING RELATIONS AND THEIR EVOLUTION}

In this section, we quantify the amount of evolution (if any) in the scaling relations between nuclear black holes and hostgalaxy properties observed locally.

We begin by showing in the left panel of Figure 5 the location of our AGNs (filled circles) in the $\log M_{\mathrm{BH}}-M_{K}$ plane. As a reference, we show there the best-fit relation derived from local inactive galaxies by Graham (2007) and given by

$$
\log M_{\mathrm{BH}}=8.29-0.37\left(M_{K}+24\right)
$$

(to guide the eye, we show also the same relation offset by \pm 0.33 dex, the total scatter in the Graham (2007) relation). Our 


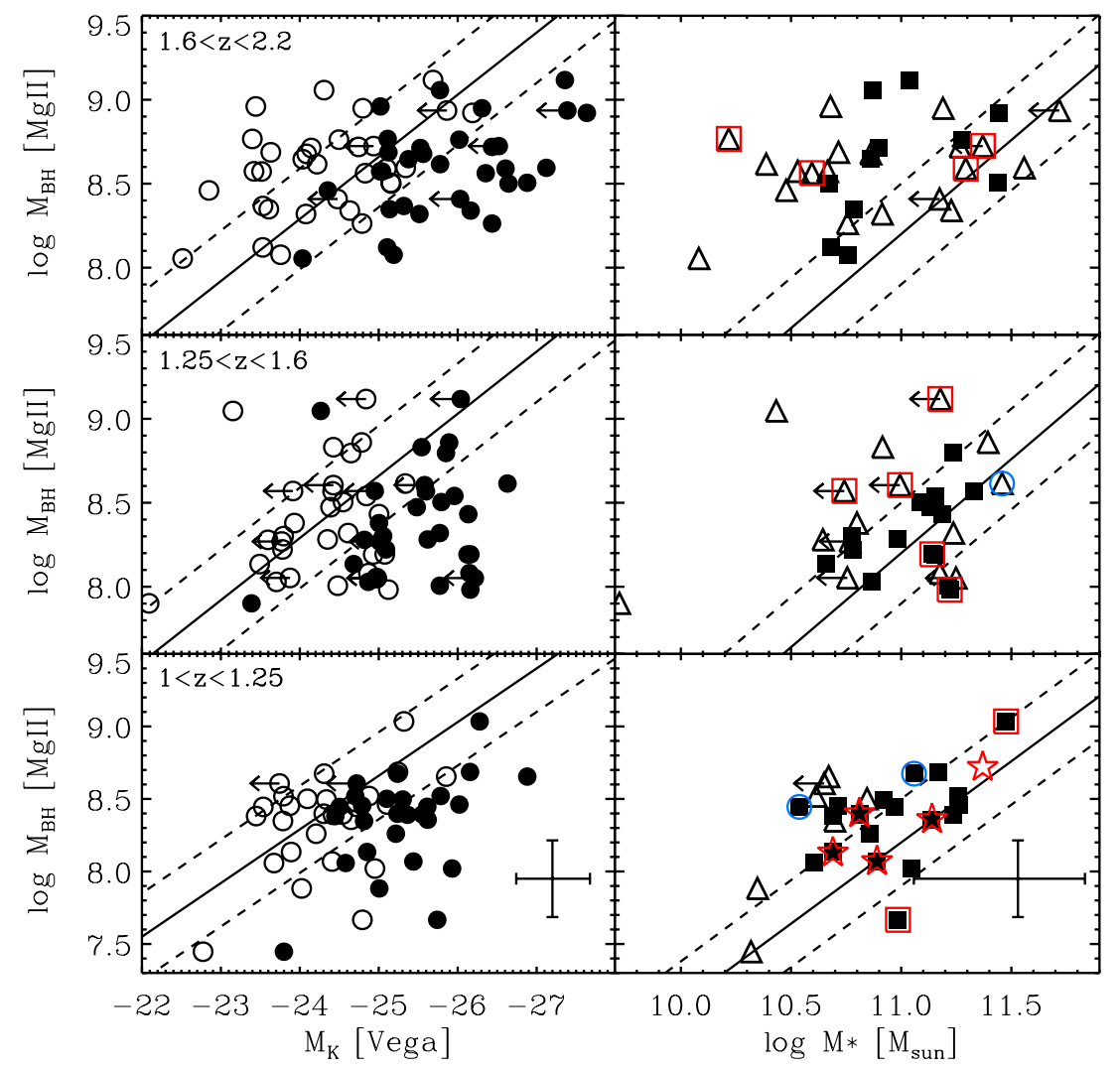

Figure 5. Scaling relations for zCOSMOS type-1 AGNs in the redshift range $1<z<2.2$. Each row shows a different redshift interval (lowest $1<z<1.25$; middle $1.25<z<1.6$; top $1.6<z<2.2$ ). Left panels: black hole mass host $K$-band absolute magnitude relation. Filled symbols represent measurements, leftward arrows upper limits on the host luminosity. The black solid line is the best fit to the Graham (2007) local spheroids sample relation, with dashed lines marking a \pm 0.3 dex offset. The typical error bars of our measurements are shown as black cross in the lower right corner. Black open circles mark the location of our galaxies when passively evolved down to $z=0$ assuming a formation redshift of $z_{f}=3$. Right panels: black hole mass host stellar mass relation. Open triangles (filled squares) denote the objects with low $(<0.33)$ and high $(>0.33)$ galaxy-to-AGN luminosity ratio in the rest-frame $K$ band, respectively. Symbols with leftward arrows represent upper limits on the host mass. The black solid line is the best fit to the Häring \& Rix (2004) local spheroids sample relation, with dashed lines marking a \pm 0.3 dex offset. Red squares mark the objects detected in the radio by VLA at $1.4 \mathrm{GHz}$, while red stars mark the location of the 5 zCOSMOS AGN in the Jahnke et al. (2009) sample. Blue circles mark the objects with absorption features in the $\mathrm{Mg}$ II line. The typical error bars of our measurements are shown as black cross in the lower right corner.

(A color version of this figure is available in the online journal.)

objects are shifted toward brighter hosts and/or smaller black hole masses with respect to the $z=0$ relation. The objects do not seem to obey any tight relation between black hole mass and $M_{K}$, although the range of $M_{\mathrm{BH}}$ probed is relatively limited. If one tried, for the sake of comparison, to fit the sample with a relation with the same slope as in Equation (3), the best-fit normalization would be shifted by $\approx 0.28 \mathrm{dex}$, and the intrinsic scatter would be as large as 0.44 dex. We have also measured the offset, $\Gamma_{K}(z)$ (here defined as the projected distance in the $\log M_{\mathrm{BH}}-$ $\log L_{\mathrm{K}}$ plane), of each observed point from the local relation and studied its evolution as a function of redshift. The best fit obtained imposing the functional form $\Gamma_{K}(z)=\delta_{1} \log (1+z)$, gives $\delta_{1}=-0.73 \pm 0.08$ (these results are unchanged if we instead adopt the $M_{K}$ derived by fitting the SED with the P07 template). The fits are performed taking into account both errors and lower limits on $\Gamma$, using a Monte Carlo approach, described in Bianchi et al. (2007).

Such an evolution is somewhat stronger than that observed by Peng et al. (2006b), where the $R$-band luminosity of 30 lensed and 20 non-lensed QSO hosts was measured based on detailed HST image modeling of $1<z<4.5$ quasars. Their QSOs apparently lie almost exactly on the same observed $M_{\mathrm{BH}}-L_{\mathrm{R}}$ relation as their $z=0$ relic counterparts. However, as already noted by Peng et al. (2006b), high-redshift galaxies shall have a different mass-to-light ratio as compared to their $z=0$ descendants, at the very least because of passive evolution of the stellar population, in the extreme case of non-star-forming galaxies. This can be simply accounted for by passively evolving the rest-frame $K$-band luminosities down to $z=0$ to allow a more direct comparison with the local relation. We have done this using once again the Bruzual \& Charlot (2003) template libraries, and assuming all objects formed all their stars in burst at $z_{f}=3$. Their local descendants (passively evolved) would have dimmed to the magnitudes indicated by the black empty circles in the right panels of Figure 5, which are now broadly consistent with the local $M_{\mathrm{BH}}-M_{K}$ relation, but slightly offset toward large black hole to host-galaxy ratios. Such a shift toward a positive offset from the local relation would in fact be even stronger if indeed the host galaxies of our AGN sample were dominated, at least statistically, by actively star-forming galaxies, as discussed earlier in Section 3.2.

This is indeed what happens when we consider the relationship between measured black hole masses and host total stellar masses, $M_{*}$, obtained from the $\mathrm{BC} 03$ fits. In the right panels of Figure 5 we show the location of 89 zCOSMOS AGN in the $\log M_{\mathrm{BH}}-\log M_{*}$ plane. In these panels, we have separated the objects on the basis of the measured $f_{\mathrm{gal}, \mathrm{K}}$ host-to-AGN luminosity ratio in the rest-frame $K$ band (with open triangles having 


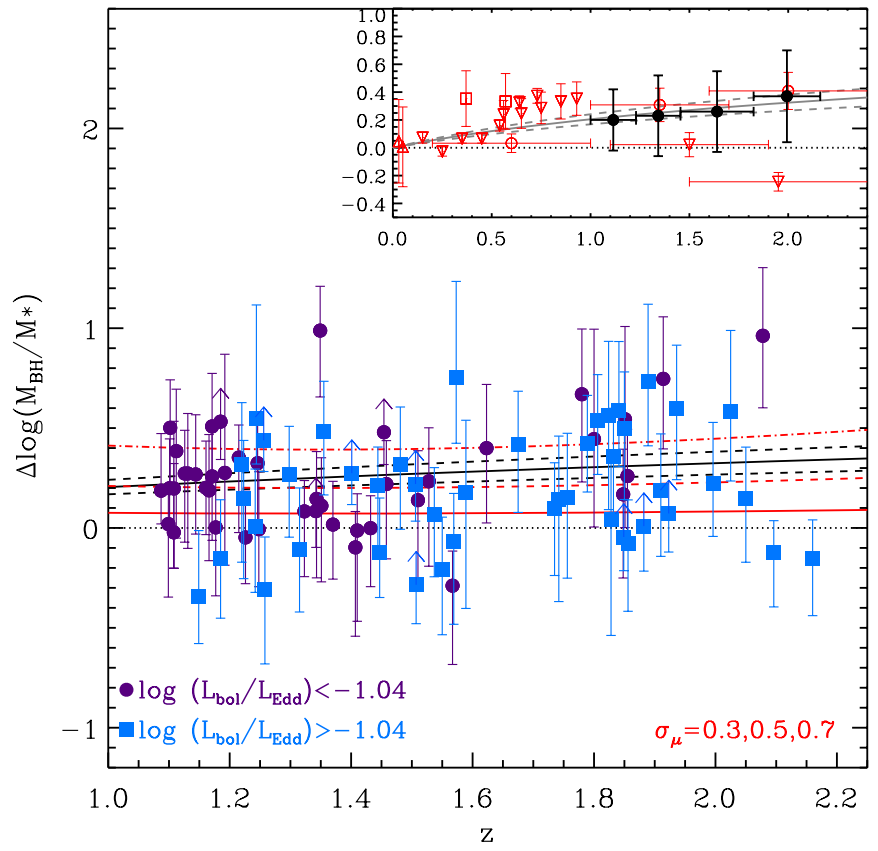

Figure 6. Redshift evolution of the offset measured for our type-1 AGN from the local $M_{\mathrm{BH}}-M_{*}$ relation. Different colors and symbols identify different ranges of Eddington ratios (purple circles $\log \left(L_{\text {bol }} / L_{\text {Edd }}\right)<-1$, and light blue squares $\left.\log \left(L_{\mathrm{bol}} / L_{\text {Edd }}\right)>-1\right)$ with upward arrows representing upper limits on the host mass. The offset is calculated as the distance of each point to the Häring \& Rix (2004) correlation. Solid black line shows the best fit obtained assuming an evolution of the form $\Delta \log \left(M_{\mathrm{BH}} / M_{*}\right)(z)=\delta_{2} \log (1+z)$; for which we found $\delta_{2}=0.68 \pm 0.12$. The red lines show the bias due to the intrinsic scatter in the scaling relation to be expected even if they are universal. Solid line is for an intrinsic scatter of 0.3 dex; dashed of $0.5 \mathrm{dex}$; dot-dashed of $0.7 \mathrm{dex}$ (see text for details). In the inset, we show a comparison of our data (black circles) with data from the literature, plotted as green open symbols: triangles are from Salviander et al. (2007, low-z) and Shields et al. (2003, high- $z$ ); squares from Woo et al. (2008) and circles from Peng et al. (2006b).

(A color version of this figure is available in the online journal.)

$f_{\text {gal }, \mathrm{K}}<0.34$ and filled squares $\left.f_{\mathrm{gal}, \mathrm{K}}>0.34\right)$. As a reference, a solid line shows the local best-fit relation between black holes and spheroids as derived by Häring \& Rix (2004):

$$
\log M_{\mathrm{BH}}=-4.12+1.12\left(\log M_{*}\right)
$$

Our objects now show a modest, but clear, offset from the local relation, especially at the high black hole mass end (see also Figure 8) and in the highest redshift bin (upper right panel of Figure 5). Radio-detected AGNs (squares) appear to be distributed similarly to the rest of the population, but low number statistics prevent us from reaching any firmer conclusion on their properties.

As our method is affected by substantial uncertainties in both black hole and host-galaxy mass, and none of the two can be treated as truly independent variable, we chose to measure the deviation of our data set from the local scaling relation by measuring the distance $\Delta \log \left(M_{\mathrm{BH}} / M_{*}\right)$ of each point from the Häring \& Rix (2004) relation, perpendicular to the relation itself. ${ }^{30}$

Figure 6 shows the measured offset of each point from the local relation as a function of redshift. The black solid

\footnotetext{
30 Given the slope in the Häring \& Rix (2004) relation $A=1.12$, the measured offset multiplied by $S \equiv \sqrt{1+A^{2}} \simeq 1.5$ gives the increase in black hole mass $\Delta M_{\mathrm{BH}}$ given a host-galaxy mass, compared to the local value. This should be kept in mind when comparing with results from previous works, which usually measure the offset from the scaling relations in terms of "excess black hole mass," i.e., vertically in Figure 5.
}

line shows the best fit obtained imposing the functional form $\Delta \log \left(M_{\mathrm{BH}} / M_{*}\right)(z)=\delta_{2} \log (1+z)$, where we find $\delta_{2}=0.68 \pm$ 0.12 . Also in this case we have used a Monte Carlo simulation to derive the best-fit linear regression coefficients; lower limits were treated as if the true value of $\Delta \log \left(M_{\mathrm{BH}} / M_{*}\right)$ were uniformly distributed up to a common value of 1.2 .

The inset of Figure 6 shows our data set and best-fit evolution, in black, as compared to a number of estimates at lower or comparable redshift. The best-fit evolution from the zCOSMOS data is in reasonable agreement with previous estimates both at lower (Salviander et al. 2007) and at higher redshifts (Peng et al. 2006b). We note here that the significant amount of scatter in our data set translates into a relatively weak statistical significance of the measured offset (see the binned points in the inset of Figure 6). Moreover, any redshift dependence within our sample only is not statistically significant. Although weaker, the evolution we measure is also marginally consistent with that observed by Treu et al. (2007) and Woo et al. (2008) from their sample of Seyfert galaxies at $z=0.36$ and $z=0.57$.

It is interesting to notice here that the majority of observational data points for the AGN sample do indeed show a broadly consistent amount of offset at all redshifts probed. This might suggest that intrinsic differences in the SMBH/host-galaxy relation between active and inactive galaxies could play an important role besides any genuine cosmological evolution. Large, uniformly selected samples of AGN hosts, spanning a larger redshift range than probed here (e.g., Decarli et al. 2009), as well as accurate comparisons of scaling relations for active (reverberation mapped) and non-active galaxies at low- $z$ (see, e.g., Onken et al. 2004), are and will be very important in disentangling true redshift evolution from other systematic differences with the local samples.

Finally, no significant trend is found by dividing our sample into fast and slow accretors on the basis of their measured Eddington ratio, contrary to the results of a number of studies of local AGNs, which have found evidence that high-accretion rate objects have smaller $M_{\mathrm{BH}} / M_{*}$ ratio as compared to less active AGNs (Greene \& Ho 2006; Shen et al. 2008; Kim et al. 2008).

\subsection{Flow Patterns in the $M_{B H^{-}} M_{*}$ Plane}

As we have mentioned before, constraining the SFR of the AGN hosts in our sample is a very difficult task, given the strong AGN component dominating the SED in the restframe optical/UV bands. Individual estimates of SFR based on the $\chi^{2}$ minimization procedure described above give results which are uncertain by up to $0.7-0.8$ dex. Nevertheless, despite these very large uncertainties on the estimated SFRs for our AGN hosts, we can try to assess the general direction of motion of the objects in the $M_{\mathrm{BH}}-M_{K}$ plane; although for each individual object it will be hard to accurately pin down the change in total stellar mass, the overall "ensemble" average motion of the flow could give interesting indications on the longer term evolution of the scaling relations.

To this end, we show in Figure 7 as red rightward-pointing arrows the predicted flow patterns of the BLAGN. The tip of each arrow marks the location where the system will find itself within $t_{\text {star }}=300 \mathrm{Myr}$, if continually forming stars at the estimated SFRs, while at the same time the central black hole keeps accreting at the measured rates for a fraction of this time equal to the AGN duty cycle $\delta_{t}$. The exact value of $t_{\text {star }}$ is of course arbitrary, and has been chosen in order to better visualize the flow pattern. Changing it, will simply rescale the length of the arrows, but will not change their orientation. For each object 


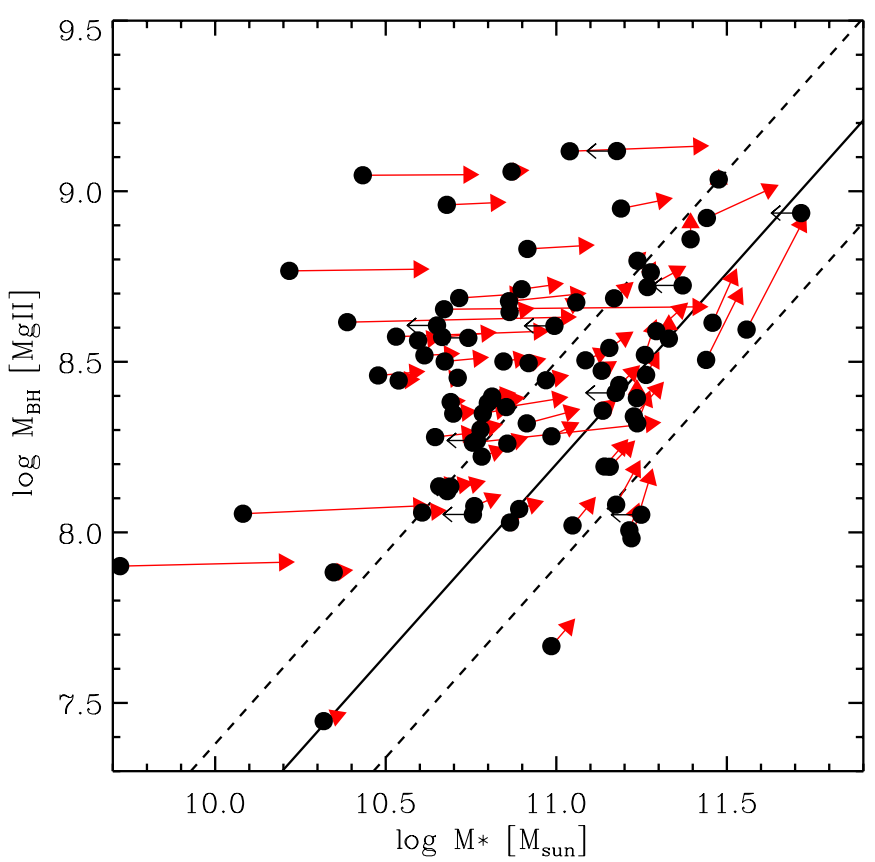

Figure 7. Black hole mass host stellar mass relation for zCOSMOS type-1 AGN in the redshift range $1<z<2$.2. Symbols with leftward arrows represent upper limits on the host mass. The black solid line is the best fit to the Häring \& Rix (2004) local spheroids sample relation, with dashed lines marking a $\pm 0.3 \mathrm{dex}$ offset. Red arrows represent the direction of evolution of the points in the $M_{\mathrm{BH}}-$ $M_{*}$ plane in $300 \mathrm{Myr}$ on the basis of their instantaneous accretion- and star formation rates and an AGN duty-cycle estimated from the amplitude of the corresponding luminosity and mass functions (see the text for details).

(A color version of this figure is available in the online journal.)

observed at redshift $z$ having nuclear bolometric luminosity $L_{\mathrm{bol}}$ and host-galaxy stellar mass $M_{*}$, the duty cycle is estimated by taking the ratio of the AGN bolometric luminosity function (Hopkins et al. 2007b) $\phi\left(L_{\mathrm{bol}}, z\right)$ to the mass function of highly star-forming galaxies (Ilbert et al. 2009), which are assumed here to represent the parent population of the sample at hand, $\phi_{\mathrm{gal}, \mathrm{HSF}}\left(M_{*}, z\right)$,

$$
\delta_{t}\left(L_{\mathrm{bol}}, M_{*}, z\right)=\frac{\phi\left(L_{\mathrm{bol}}, z\right)}{\phi_{\mathrm{gal}, \mathrm{HSF}}\left(M_{*}, z\right)} .
$$

As the mass functions have a sharp exponential cutoff at high masses, as opposed to the power-law decline of the QSO luminosity function, the duty cycle defined above increase quickly for the most massive hosts, causing the upward turn of the flow pattern. Intriguingly, the predicted motion of the objects in the $M_{\mathrm{BH}}-M_{*}$ plane does lead to a reduced scatter of the points, i.e., the flow appears to be "converging." To give a simple quantitative estimate of this effect, we measure the scatter by fitting all points (apart from those with only upper limits for the host-galaxy properties) with a linear relation. Given the reduced dynamic range in black hole masses we are probing, and for the sake of simplicity, we fix the slope of the correlation to the locally measured one (1.12; Häring \& Rix 2004), and let the normalization be a free parameter. The observed points have thus a normalization of about -3.7 and an intrinsic scatter of 0.43 . The tips of the arrows, instead, move closer to the local relation (normalization $\approx-3.9$ ) and with a much reduced scatter of 0.34 .

Future observations of lower redshift AGNs, for which an accurate determination of both total stellar mass and SFR is more easily achieved by a combination of multi-band photometry and high-resolution spectroscopy will provide much better (and more reliable) maps of the flow patterns of the AGN-hostgalaxy systems, revealing fundamental details on their physical coupling.

\section{SYSTEMATICS AND SELECTION EFFECTS}

The main result of the previous section is that our estimates of the type-1 AGN host physical parameters are (although marginally) inconsistent with the hypothesis that they lie on the $z=0$ scaling relation. Here we wish to discuss how much of this observed offset can be due to various systematics and selection effects. We identify here two kinds of biases: the first is the combination of systematics inherent to our methods to measure either BH or host galaxy's mass, and we will discuss these first (Sections 6.1, 6.2, and 6.3). The second, more subtle, is a "luminosity/mass function weighted" bias on any AGNselected sample introduced by the intrinsic scatter in the scaling relations (Adelberger \& Steidel 2005; Fine et al. 2006; Lauer et al. 2007; Treu et al. 2007). This could induce a spurious effect on the measured offsets, provided the scatter in the relation is large enough. We will discuss this effect in some detail in Section 6.4.

\subsection{IMF and Galaxy Stellar Masses}

In this work, mainly to allow a direct comparison with most previous works on the subject, we have adopted a Salpeter IMF to calculate the total stellar mass of the host galaxies based on the BC03 SED fitting procedure. As mentioned already in Section 3, adopting a different IMF will result in a systematic shift of the best values for $M_{*}$, typically reducing the stellar mass, and increasing the ratio $M_{\mathrm{BH}} / M_{*}$. To quantify the systematic uncertainty in the measured evolution introduced by the uncertainty in the IMF, we have re-calculated the stellar masses using a Chabrier IMF. We thus shifted the estimated values of the total stellar mass of the AGN hosts by -0.255 dex (Pozzetti et al. 2007) and found the following values for the exponent of the redshift evolution function: $\delta_{2}=1.15 \pm 0.13$. The smaller host masses implied by the new choice of IMF result in a larger positive offset of the $M_{\mathrm{BH}} / M_{*}$ ratio from the locally determined value, requiring a more pronounced evolution. On the other hand, it should be kept in mind that realistic SFHs can be different from the smooth ones adopted here, due to the presence of short bursts of star formation. In fact, Pozzetti et al. (2007) have tested how the measured stellar masses change when random bursts are superimposed on smooth SFHs (with a similar range of combinations of $\tau$ and $t_{\text {age }}$ to the one chosen here). They conclude that, for $z>1.2$ samples, the mean mass computed with smooth SFH is on average 0.16 dex smaller than the one computed using a bursty SFH (see Figure 5 in Pozzetti et al. 2007). This offset is smaller than our typical errors on the stellar masses. Moreover, a robust assessment of the "burstiness" of the SFH cannot be performed on our sample, as the number of extra parameters required (duration of a burst, fraction of stellar mass produced in the burst, time since last one) would surely introduce strong degeneracies in our fits.

Another well-known source of systematic uncertainty lies in the choice of the $\mathrm{BC} 03$ templates to describe the stellar populations of the AGN hosts. Different groups have in recent years built different stellar population models using different treatments of stellar structure and evolution (see, e.g., Fioc \& Rocca-Volmerange (1997), Silva et al. (1998), Maraston 
(2005)). A thorough discussion of the relative differences among them is far beyond the scope and the aims of this paper (but see, e.g., Longhetti \& Saracco 2009). Suffice it to say, in this context, that the typical offset in the estimated masses of test galaxies with ages similar to those considered here are much smaller (of the order of $\pm 0.1-0.15$ dex; see, e.g., Cimatti et al. 2008; Longhetti \& Saracco 2009) than the statistical uncertainties of our mass measures.

Finally, it is clear that by comparing total stellar masses of AGN hosts with the bulge/spheroid masses of the local galaxies originally used to derive and identify the scaling relations, we are introducing a significant bias (see also Jahnke et al. 2009; Bennert et al. 2009). Lacking any imaging information and reliable bulge-to-disk (B/T) decomposition, we can just argue that, at the very least, the black hole to bulge mass ratio should show an even larger offset from the local scaling relation. Systematic trends in the B/T ratio with redshift (see, e.g., Merloni et al. 2004) will need also to be taken into account.

\subsection{Black Hole Mass Measurements}

We have already mentioned in Section 4 that there is currently a substantial uncertainty on the actual parameter of the virial relationship to be used for the estimate of black hole masses in BLAGNs. In the calculations so far, we have adopted the McGill et al. (2008) expression, but different relationships, based on different calibrations and/or assumptions about the BLR geometry exist in the literature.

To test the systematic effects on the measured evolution of the black hole to host-galaxy mass ratio evolution, we have re-calculated black hole masses using the MLD04 and V09 relations (see Section 4).

Adopting the relation of McLure \& Dunlop (2004), which has a steeper BLR size-luminosity relation (0.62 instead of $0.5)$, substantially reduces the amount of observed evolution: we obtain, for the exponents of the redshift evolution function, $\delta_{2}=0.47 \pm 0.12$, bringing it closer to the expectations of a purely luminosity-bias dominated effect if the $M_{\mathrm{BH}}-M_{*}$ relation has an intrinsic scatter as large as $0.5 \mathrm{dex}$ (see section below). On the other hand, adopting the V09 relation, which produce, on average, larger black hole masses than in our fiducial case, we obtain $\delta_{2}=0.91 \pm 0.12$, indicating a larger amount of positive evolution. It is worth keeping in mind, however, that the McLure \& Dunlop (2004) normalization of the "virial" black hole mass estimate is based on a specific (theoretically motivated) assumption on the BLR geometry, while the V09 one is empirically calibrated with the local $M_{\mathrm{BH}}-\sigma$ relation, i.e., it does not assume any preferred geometry.

Other possible systematic effects could be due to evolution of the physical properties of the BLR itself. However, no significant trend with redshift was found in our data for either the Fe II emission strength (measured relative to the continuum luminosity), or the overall goodness of the continuum fits, that could have signaled an inadequacy in our model of the $\mathrm{Fe}$ II template (due, for example, to systematic changes in metallicity). Higher signal-to-noise spectra are probably needed to assess these issues with the due care.

Finally, we would like to note here that, when comparing with results present in literature, one should take into account the different methods of line fitting and $\mathrm{Fe}$ II subtraction. A thorough analysis of the induced bias is not straightforward, as the specific techniques of broad-line fitting can be different. Nevertheless, we have ourselves performed a new fit of the $\mathrm{Mg}$ II line complex without including any Fe II template in the continuum. The distribution of the (log of the) ratio between the FWHM calculated without and with Fe II subtraction is centered at a positive offset of about 0.1 dex (which would correspond to a difference in mass of about $0.2 \mathrm{dex}$ ), but with a significant tail of higher ratios toward low values of the FWHM.

\subsection{Choice of AGN SED}

In Section 3, we have shown how the measure of the restframe $K$-band luminosity of the BLAGN hosts does not depend strongly on the set of galaxy SED templates used to fit the composite AGN+galaxy SED, provided that the same AGN SED is used (we adopt here the Richards et al. (2006) mean QSO SED, allowing for additional dust extinction of the nuclear light).

An obvious possible objection to our results is that, if the AGN SED were markedly different from the Richards et al. (2006) template at the typical luminosities of the zCOMSOS bright sample, we would be introducing a severe bias in our measures of the host-galaxy parameters. More worryingly, any systematic trend whereby AGN SEDs change with either redshift, luminosity, black hole mass, or Eddington ratio, would introduce spurious trends in the measured evolution of the offset from the local scaling relations.

Elvis et al. (2009, hereafter E09) performed a thorough, systematic study of the SED of the X-ray (XMM-COSMOS) selected, spectroscopically confirmed, type-1 (broad-line) AGNs in the COSMOS field. The interested reader is referred to E09 for a discussion of the main properties of the sample. Here we would like to point out that, even when selecting only those objects that are classified as "pointlike" from their ACS images, in the redshift range of interest here $(1<z<2.2)$ a non-negligible contribution due to the compact stellar emission from the host is still significantly present in the observed SED, even in the brightest sources.

Indeed, the mean SED of the "pointlike" type-1 COSMOS AGNs with $L_{\text {bol }}>10^{45.5}$ shows a less pronounced dip in the $1 \mu \mathrm{m}$ region than either the Elvis et al. (1994) or the Richards et al. (2006) templates. As a test, we have used this new, COSMOS based, AGN SED together with the BC03 templates to fit the composite SED of the objects in our sample. As expected, the flatter mean AGN SED results in a lower residual host-galaxy contribution. The number of "undetected" hosts (i.e., they have only upper limits in the rest-frame $K$-band magnitude estimate) rises from 10 to 24 ; on average, the hosts result about $0.4-0.5$ magnitudes fainter. Consequently, the positive offset of type-1 AGN from the local scaling relations would be larger (we have measured, for these choice of AGN $\mathrm{SED}, \delta_{2} \simeq 1.3$ ).

We argue that, on the basis of the photometric data only, it is not possible to decide whether the apparent flatness of the AGN SED at NIR wavelengths is indeed due to a dramatic change of intrinsic nuclear continuum emission (thus minimizing the host-galaxy contribution), or, in contrast, it is mainly due to stellar light contamination. As the QSO SEDs we have adopted represent an extreme within the COSMOS database (see E09), our approach, which maximizes the host's contribution is the most conservative one with respect to the measured evolution of the $M_{\mathrm{BH}} / M_{*}$ ratio. Independent clues on the intrinsic nuclear continuum shape from, e.g., detailed spectroscopic and/or polarimetric studies will be helpful.

Finally, we have also considered the effects of AGN variability on the observed SED. This has been studied in detail by Salvato et al. (2009), where it was demonstrated that 
correcting for even relatively small variation of the photometric points due to the non-simultaneous observation times can significantly improve the photometric redshift determination for AGNs. A "variability corrected" photometric catalog is available for the subsample of X-ray detected type-1 AGNs (82/89 objects in our sample). We have thus recomputed the hosts total stellar masses and studied the evolution of the $M_{\mathrm{BH}} / M_{*}$ ratio also for them, finding no significant difference in the redshift dependence of the measured offset $\left(\delta_{2}=0.84 \pm 0.13\right)$.

\subsection{Selection Bias}

In this section we estimate the possible bias due to selection effects. Our objects are selected essentially on the basis of the nuclear (AGN) luminosity, and on the detectability of the broad Mg II emission line, clearly leading to a bias toward more massive black holes, similar to Malmquist (1924) bias for luminosity-selected samples of standard candles.

In fact, there is a more subtle effect, generally applicable to all cases where two properties of a class of objects are known to be correlated with certain intrinsic dispersion, and one wishes to determine the probability distribution of one of the two quantities, having selected objects on the basis of measurement of the other one. For purely flux limited samples, this bias was already discussed and calculated by Kellerman (1964, see the appendix), for the case in which the two quantities were the spectral indices of the AGN at two different radio frequencies. This was then generalized by Francis (1993) to the case of AGN spectral slopes in any two given independent bands. For the specific case of black hole and host-galaxy masses (or velocity dispersion), this bias was discussed already in Adelberger \& Steidel (2005), but has been scrutinized in depth in Lauer et al. (2007), which also recovered the Kellerman (1964) results for the specific case of a flux limited sample, and we refer the curious reader to the Lauer et al. (2007) paper for a more thorough discussion.

Here we make use of the main analytic results of Lauer et al. (2007) and apply them to our particular selection criteria. The null hypothesis we put under test is that the local scaling relation between black hole and host-galaxy mass, assumed here to be given by Equation (4), with an intrinsic scatter $\sigma_{\mu}$, does not change with redshift, neither in normalization and slope, nor in scatter.

It is in fact the intrinsic scatter in the local relation, together with the observed shape of the mass and/or luminosity function of the selected objects in the appropriate redshift ranges that determine the bias. In a nutshell, cosmic scatter in the $M_{\mathrm{BH}}-M_{*}$ relation implies that there is a range of masses $\log M_{*} \pm \sigma_{\mu}$ for each object of a given black hole mass $M_{\mathrm{BH}}$, where we have assumed, for simplicity, a symmetric scatter in the relation. If the number density of galaxies is falling off rapidly in the interval $\log M_{*} \pm \sigma_{\mu}$, it will then be more likely to find one of the more numerous small mass galaxies associated with the given black hole, and therefore a larger ratio $M_{\mathrm{BH}} / M_{*}$. Thus, given a distribution of galaxy masses (mass function $\phi\left(\log M_{*}\right)$ ), and provided that the scatter $\sigma_{\mu}$ is not too large, the logarithmic offset of each point from the correlation (4), assumed to be held fixed to the local determination, is given by (cf. Lauer et al. (2007), Equation (14)):

$$
\begin{aligned}
\Delta \log \left(M_{\mathrm{BH}} / M_{*}\right) & =(1 / S) \times \Delta \log M_{\mathrm{BH}} \\
& \approx \sigma_{\mu}^{2}\left(\frac{d \log \phi}{d \log M_{*}}\right)_{\log M_{*}=(\mu-A) / B},
\end{aligned}
$$

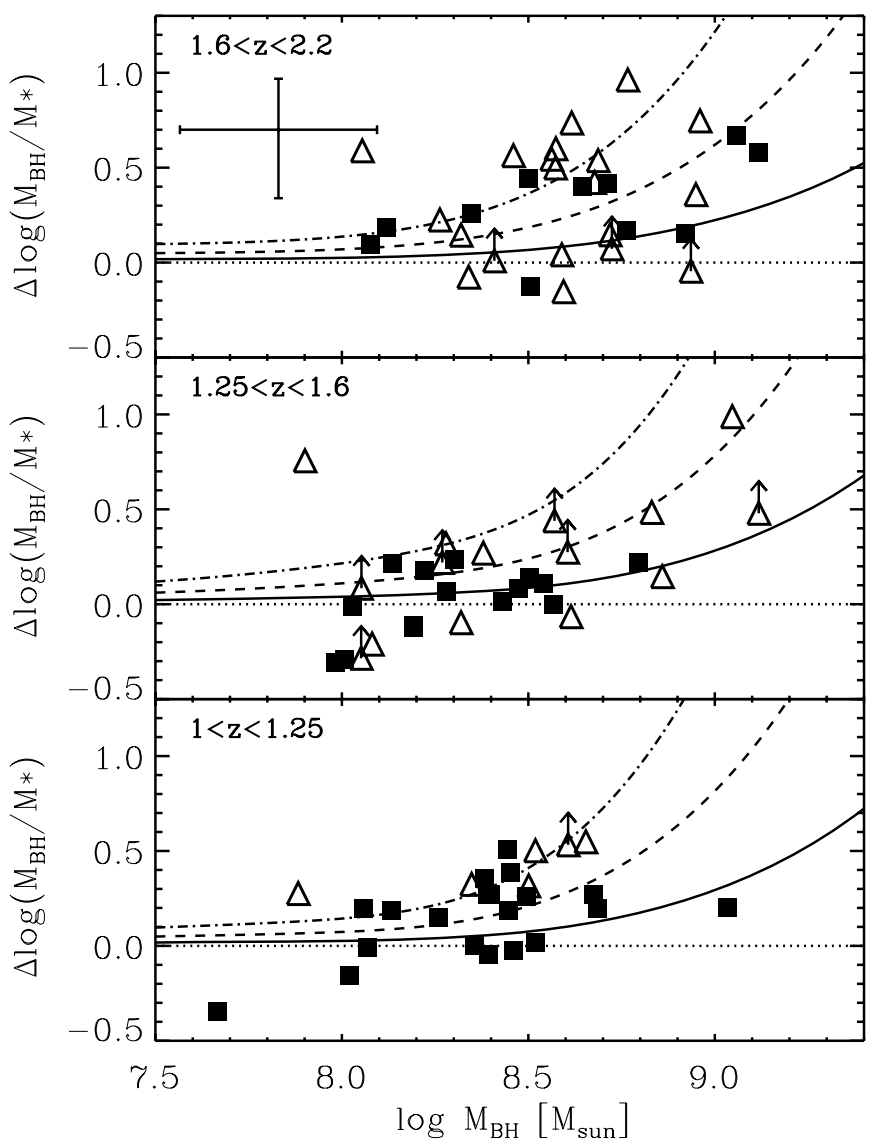

Figure 8. Offset from the local $M_{\mathrm{BH}}-M_{*}$ relation as a function of black hole mass. Filled symbols represent measurements, symbols with upward arrows represent upper limits on the host mass. Open triangles (filled squares) denote the objects with low $(<0.33)$ and high $(>0.33)$ Galaxy to AGN luminosity ratio in the rest-frame $K$ band, respectively. Lines show the bias due to the intrinsic scatter to be expected even if the local relation is universal. Solid lines are for an intrinsic scatter of $0.3 \mathrm{dex}$, dashed of $0.5 \mathrm{dex}$, and dot-dashed of $0.7 \mathrm{dex}$. The typical error bars of our measurements are shown as black cross in the upper left corner.

where $1 / S=0.67$ (see footnote 30 in Section 5), $\mu=\log M_{\mathrm{BH}}$ and $(A, B)=(1.12,-4.12)$ are slope and intercept of the relation (4). We estimate the logarithmic derivative of the mass function $\frac{d \log \phi}{d \log M_{*}}$ using the mass function determination from the S-COSMOS galaxy survey (Ilbert et al. 2009; masses have been recalculated to adjust to our choice of Salpeter IMF) in the same redshift range probed by our BLAGNs. Figure 8 shows our objects in the offset-black hole mass plane, where solid lines mark the expected bias from Equation (6) in three different redshift ranges, in the case of $\sigma_{\mu}=0.3$. Dashed and dot-dashed lines, instead, correspond to the cases of $\sigma_{\mu}=0.5$ and 0.7 , respectively. This plot clearly shows that the offset we measure is in excess to what expected in the most extreme case of large intrinsic scatter in the local relation, estimated by Novak et al. (2006) to be less than 0.5 dex (see also Gütelkin et al. 2009). This is mainly due to the fact that the AGN black hole masses we measure at the depth of the zCOSMOS selection function are not extremely large, and correspond, according to Equation (4) to a range of host-galaxy masses where the mass function is not falling off too steeply. On the other hand, our observations could be explained in terms of luminosity bias only if the scatter in the $M_{\mathrm{BH}}-M_{*}$ relation were as large as $0.5-0.7$ at $z>1$.

Yet another test is possible, however. When studying the redshift evolution of $\Delta \log \left(M_{\mathrm{BH}} / M_{*}\right)$, fitting its redshift dependence 
with a functional form (Section 5), one does effectively take the average of the offset over a range of AGN luminosities above a well determined selection cut in any given redshift bin. In this case, it can be shown (Lauer et al. 2007, Equation (25)) that the average offset at any given redshift is given by

$$
\left\langle\Delta \log \left(M_{\mathrm{BH}} / M_{*}\right)\right\rangle(z)=\frac{0.67 \sigma_{\mu}^{2}\left[\Psi\left(L_{\min }, z\right)-\Psi\left(L_{\max }, z\right)\right]}{\int_{L_{\min }}^{L_{\max }} \Psi(L, z) d L},
$$

where $\Psi(L, z)$ is the type-1 AGN luminosity function at redshift $z$ and $L_{\min }$ and $L_{\max }$ are the minimum and maximum luminosities of the AGNs that can enter our sample at the same redshift, given our survey selection function. We have calculated this bias adopting the VVDS type-1 AGN luminosity function of Bongiorno et al. (2007), and the results are plotted as red lines in Figure 6. For our type-1 AGN sample, which extends well below the knee of the type-1 luminosity function in this redshift range, the expected bias in the offset is almost constant with redshift, amounting to $\approx 0.3(0.1)$ dex for $\sigma_{\mu}=0.5(0.3)$. This is consistent with the estimate for $\Delta \log M_{\mathrm{BH}}$ of Lauer et al. (2007) based on the local AGN luminosity function of Boyle et al. (2000), and slightly smaller than the measured offset. On the other hand, a larger intrinsic scatter in the scaling relation at the redshift of interest (up to $\sigma_{\mu}=0.7$, dotted lines in Figure 6) could indeed be the cause of the measured offset in our sample.

A note of caution is however in place. Our measured offset is based on a black hole mass "virial" estimator that has been implicitly calibrated on the local $M_{\mathrm{BH}}-\sigma_{*}$ relation (Onken et al. 2004). This is usually done with the justification that the true geometry of the BLR is not known, which enters as a multiplicative factor in the virial relation. However, the reverberation-mapped AGN used by Onken et al. (2004) to carry out this normalization, could themselves be affected by the "luminosity/mass function weighted bias." If that were the case, and if the local AGN samples spanned a similar mass and luminosity range as ours, the bias would be much reduced, and possibly canceled out completely (as suggested also by the results of Kim et al. 2008). The selection function of the Onken et al. (2004) AGN is, however, far from being well understood, and a quantitative estimate of the true expected residual bias is beyond the scope of this work. As we discussed above, large, uniformly selected samples of AGN hosts, spanning a larger redshift range than probed here, as well as accurate comparisons of scaling relations for active (reverberation mapped) and nonactive galaxies at low- $z$ with similar selection functions, are and will be very important in disentangling true redshift evolution from other biases and systematic differences with the local samples.

Finally, we would like to mention the further aspect of what, in general terms, can be defined the luminosity bias of the AGN, namely the fact that faint BLAGNs cannot be detected in bright galaxies. This is clearly an important issue to consider in our case, given this zCOSMOS sample has a larger fraction of faint AGNs.

In order to assess this, we have divided sources in Figures 5 and 8 according to the measured luminosity ratio in the restframe $K$ band, with open triangles (filled squares) marking the most (least) AGN-dominated sources. It is interesting here to point out that, indeed, high contrast (AGN-dominated) objects do seem to slightly bias the result toward high $M_{\mathrm{BH}} / M_{*}$ ratios. However, removing all such objects, the main results presented here of a redshift evolution of the scaling relation, are not only confirmed, but do appear to be strengthened, as AGN-dominated objects (open triangles) are the largest outliers also in the lowest redshift bin.

\section{DISCUSSION AND CONCLUSIONS}

\subsection{Our Results}

We have used an AGN+host-galaxy SED decomposition technique to infer the physical properties of the hosts of 89 (moderately luminous: i.e., mostly sub- $L *$ ) type- 1 AGN in the zCOSMOS survey. Thanks to the deep, intensive multiwavelength coverage of the COSMOS field, the observed SEDs are sampled to such a degree that the decomposition technique works reasonably well. We are thus able to derive rest-frame $K$-band magnitudes and total stellar masses for the majority of our sample (80\%-90\%, depending on the choice of AGN SED). Noticeably, our method allows us to properly quantify the uncertainty in each measurement.

The bulk of the sample of BLAGN hosts we have studied have total stellar masses in the range $10^{10.5}-10^{11.3} M_{\odot}$. Both the derived mass-to-light ratios and the sample average SFRs seem to suggest that they are moderately-to-highly star-forming objects, in good agreement with the host properties of both type- 1 and type-2 (obscured) X-ray- and/or IR-selected AGN, either in a lower (Kauffmann et al. 2003; Jahnke et al. 2004; Hickox et al. 2009; Silverman et al. 2009) and in a similar (Brusa et al. 2009; Ross et al. 2009) redshift range. Reassuringly, also the fits with the P07 phenomenological templates indicate an overall preference for star-forming hosts over passive (elliptical) ones. Obviously, a more accurate determination of the individual SFRs is hampered by the dominant contribution of the AGN in the rest-frame UV/Optical part of the spectra.

The black hole masses derived with the "virial" or "empirically calibrated photo-ionization" method are broadly distributed around $\log M_{\mathrm{BH}} \sim 8.5$. Interestingly, the mass range probed by our sample corresponds to that where the most stringent constraints on the $z=0$ scaling relations are available. The Eddington ratios $\lambda$ of our objects have a mean of $\log \lambda \approx-1$. We observe a clear trend between Eddington ratio and bolometric luminosity, that could be indicative of some specific luminositydependent AGN lifetimes distribution (Hopkins \& Hernquist 2009), but the underlying effect of various selection biases in determining this trend needs to be further assessed (Trump et al. 2009).

For a large number of objects in the sample (68/89; but the number falls to $24 / 89$ if we consider the errors) the measured black hole to stellar host mass ratio is positively offset from that predicted by the local Häring \& Rix (2004) local scaling relation. Assuming a redshift-dependent evolution in the $\Delta \log \left(M_{\mathrm{BH}} / M_{*}\right)$ of the form $\delta_{2} \log (1+z)$, we measure $\delta_{2}=0.68 \pm 0.12_{-0.3}^{+0.6}$, where the large asymmetric systematic errors stem from the uncertainties in the hosts' IMF, in the calibration of the virial relation used to estimate $\mathrm{BH}$ masses and in the mean QSO SED to be used.

The scatter in the measured offset is substantial at all redshifts probed, such that we could still be consistent with a lack of evolution in the scaling relation at the $2 \sigma$ level, as shown by the inset of Figure 5. There, it is also apparent that the majority of observational data points for AGN samples in the published literature do indeed show a broadly consistent amount of offset at all redshifts probed. This might suggest that intrinsic differences in the SMBH/host-galaxy relation between active and inactive galaxies could play an important role besides any genuine cosmological evolution. 
Jahnke et al. (2009) have independently computed total stellar masses for a small sample (18 objects) of X-ray-selected AGNs in the COSMOS field for which simultaneous HST/ACS and HST/NICMOS observations allow us to clearly image the resolved hosts. The two independent methods for measuring stellar masses are in broad agreement with each other, with a dispersion well within the (large) uncertainties that characterize each of these methods. Five out of 18 of them have also zCOSMOS spectra, and are part of the sample described here (empty stars in Figure 5). However, the objects in the Jahnke et al. (2009) sample do not show any significant offset from the local scaling relation. This might be due to a statistical fluctuation (more so given that they tend to lie in the lower redshift range probed by our sample, where the offset from the local scaling relation is smaller), or may indicate a more serious issue with the different estimates of the mass-to-light ratio of the AGN hosts. Larger samples of HST-imaged AGN hosts at longer wavelengths (with the newly installed WFC3) will be extremely important to settle this issue and, in general, to improve the calibration of our SED-based method to estimated host-galaxy masses for AGNs.

We have taken particular care in examining the effects of the bias inevitably introduced by any intrinsic scatter in the $\mathrm{BH}$ host mass scaling relation into any AGN-selected sample, as ours. We conclude that our data cannot possibly be explained if type-1 AGNs and their hosts at $1<z<2$ lie on a scaling relation which has the same slope, normalization, and scatter as the locally observed one. On the other hand positive evolution of the average $M_{\mathrm{BH}} / M_{*}$ ratio (larger black holes at early times in unobscured AGNs) or of the intrinsic scatter (or a combination of the two) are needed to explain our results.

\subsection{Implications for Theoretical Models}

What are the implications of these findings for our understanding of the cosmological co-evolution of black holes and galaxies? Let us briefly discuss recent theoretical investigations on this issue and the corresponding predictions for the evolution of the scaling relations.

One of the earliest SAMs to incorporate the evolution of SMBHs and the associated feedback effect were those by Granato et al. (2001, 2004). There, triggering of AGN activity is not directly linked to merger activity, but rather generically to the process of bulge/spheroid formation. The rate of star formation and black hole accretion is regulated by the starlight radiation drag, and consequently, in a typical system the ratio $M_{\mathrm{BH}} / M_{*}$ is initially small and rapidly grows until the AGN feedback sweeps the remaining gas. QSOs and, in general, type-1 AGN are thus associated with the final stage of bulge formation, and it is very hard to produce any positive offset from the local relation like the one we measure.

This is however a problem common to all feedback models in which the black hole energy injection is very fast (explosive). Indeed, the first published predictions of merger-induced AGN activity models (Robertson et al. 2006) indicated that, if strong QSO feedback is responsible for rapidly terminating star formation in the bulge (Di Matteo et al. 2005; Springel et al. 2005), then very little evolution, as well as very little scatter, is expected for the scaling relations. However, later works within the same theoretical framework (Hopkins et al. 2007a; Hopkins et al. 2009) have analyzed in greater depths the role of dissipation in major mergers at different redshifts. Under the assumption that black hole and spheroids obey a universal "black hole funda- mental plane" (BHFP), where $M_{\mathrm{BH}} \propto M_{*} \sigma_{*}^{2}$, they show how, in gas-richer environments (at higher redshift), dissipation effects may deepen the potential well around the black hole, allowing it to grow above the $z=0 M_{\mathrm{BH}}-M_{*}$ relation, to a degree marginally consistent with our results. However, in the same physical framework, the $M_{\mathrm{BH}}-\sigma_{*}$ relation is almost independent on redshift, which would contradict the observational results of Woo et al. (2006); Treu et al. (2007); Woo et al. (2008).

Another, related effect was discussed in Croton (2006). There it was assumed that major mergers can trigger both star formation in a bulge as well as black hole growth, in a fixed proportion. However, bulges can also acquire mass by disrupting stellar discs, a channel that should not contribute to black hole growth. The relative importance of these two paths of bulge formation may lead to lighter bulges for a given black hole mass at high redshift, as disks have a smaller stellar fraction. A subsequent study of this and other dynamical process of diskto-bulge transformation was included in the work by Fontanot et al. (2006) and Malbon et al. (2007). They also confirmed qualitatively the predictions of Croton (2006), but found a much smaller effect, at most a factor $\sim 2$ at $z=2$ in the Malbon et al. (2007) work, and preferentially for small mass black holes $M_{\mathrm{BH}} \lesssim 10^{8} M_{\odot}$. Even more complex SAMs including various flavors of AGN-driven winds and their feedback effects (Fontanot et al. 2006) can lead to various degrees of positive redshift evolution of the average $M_{\mathrm{BH}} / M_{*}$ ratio (Lamastra et al. 2009).

As a general rule, we observe that, following increasing observational efforts to study the evolution of scaling relations, SAMs have become more sophisticated over the years. This increase of sophistication has allowed more complex behaviors of the coupled black holes-galaxy systems over cosmological times. As it is expected, more complex models also lead to an increase in the predicted scatter, even though a clear theoretical study on the redshift evolution of such scatter is still missing (but see the recent attempts by Lamastra et al. 2009 and Somerville 2009).

Hints from hydrodynamical simulations, both of isolated mergers (Johansson et al. 2009) and of relatively small cosmological boxes (Coldberg \& di Matteo 2008) do indeed show a large scatter in the instantaneous ratio between black hole accretion and SFRs, similar to what was found here (see also Silverman et al. 2009), thus suggesting that on the relatively short timescales over which un-absorbed AGNs/QSOs are visible the physical connection between black holes growth and galaxy formation must be complex, too. How this would impact on the statistical and evolutionary properties of the galaxy population as a whole, however, is far from clear.

The results we have presented in Section 5, coupled with analysis of selection biases of Section 6.4, would suggest that a greater effort should be made by theoretical modellers to include a more accurate and realistic study of the evolution of the intrinsic scatter in any scaling relations, as well as that of slope and normalization.

The "increased scatter" hypothesis, as an explanation of the observed offset, and its inevitable consequence that a "luminosity function weighted" bias plays a significant role in the observed evolution of scaling relations, could be strengthened if recent claims of undermassive black holes in IR-selected galaxy samples were confirmed (Shapiro et al. 2009). Indeed, we should expect that in samples selected purely on the basis of the hostgalaxy stellar mass, rather than on AGN properties, the intrinsic scatter in the scaling relation should produce a bias going in the 
opposite direction as those discussed above, depending on the exact shape of the $\mathrm{BH}$ mass function at the redshift considered.

\subsection{Concluding Remarks}

By taking advantage of the unique combination of VLT spectroscopy and deep multi-wavelength coverage of the COSMOS field, we have presented here a novel method to study the physical link between SMBHs and their host galaxies in type-1 (unobscured) AGNs in the crucial redshift range $1 \lesssim z \lesssim 2$. The main focus of this work is on the capability of our SED decomposition technique to provide reliable estimates of the total stellar mass of the AGN hosts, and, even more importantly, reliable estimates of its uncertainty.

The main result of our study is the observation of an offset in the $M_{\mathrm{BH}}-M_{*}$ relation, such that, in the redshift range probed, for their given hosts black holes are on average 2-3 times larger than their counterparts in the nuclei of nearby inactive galaxies. A thorough analysis of all possible observational biases induced by intrinsic scatter in the scaling relations reinforces the conclusion that an evolution of the $M_{\mathrm{BH}}-M_{*}$ relation must ensue for actively growing black holes at early times: either its overall normalization, or its intrinsic scatter (or both) must increase significantly with redshift.

We close with two recommendations for future studies of the subject. From the observational point of view, it will be very important to explore methods to derive robust black hole mass estimates in high-redshift samples of obscured AGNs, that can be selected purely on the basis of their host-galaxy properties. Broad emission lines at longer wavelengths, where the effect of obscuration are less severe, could be very useful in this respect. Also, a better understanding of the differences in the hosts' properties of active and inactive black holes is needed to allow a more meaningful comparison with the local scaling relations, and a better assessment of their evolution. From the theoretical point of view, more efforts should be devoted to derive robust predictions for the coupled evolution of slope, normalization, and intrinsic scatter in the scaling relations, and to properly include in the models the selection effects that clearly play an often decisive role in the observational studies of co-evolving galaxies and black holes.

This research was supported by the DFG cluster of excellence "Origin and Structure of the Universe." We thank the anonymous referee for carefully reading the manuscript and providing us with insightful and constructive remarks. We also thank R. Decarli, F. Fontanot, O. Gerhard, J. Greiner, P. Hopkins, S. Komossa, N. Menci, P. Monaco, H. Netzer, R. Saglia, L. Wisotzki, J.-H. Woo for their useful comments and suggestions. The HST COSMOS Treasury program was supported through NASA grant HST-GO-09822. We thank Tony Roman, Denise Taylor, and David Soderblom for their assistance in planning and scheduling of the extensive COSMOS observations. We gratefully acknowledge the contributions of the entire COSMOS collaboration consisting of more than 80 scientists. More information on the COSMOS survey is available at http://www.astro.caltech.edu/ cosmos. It is a pleasure to acknowledge the excellent services provided by the NASA IPAC/ IRSA staff (Anastasia Laity, Anastasia Alexov, Bruce Berriman, and John Good) in providing online archive and server capabilities for the COSMOS data sets. In Italy, this work was partly supported by an INAF contract PRIN/2007/1.06.10.08 and an ASI grant ASI/COFIS/WP3110 I/026/07/0; in Mexico by the CONACyT grant program 83564 and PAPIIT IN110209; in
Germany by the Bundesministerium für Bildung und Forschung/Deutsches Zentrum für Luft und Raumfahrt and the Max Planck Society.

\section{REFERENCES}

Adelberger, K. L., \& Steidel, C. C. 2005, ApJ, 627, L1

Arnouts, S., et al. 2007, A\&A, 476, 137

Bell, E. F., McIntosh, D. H., Katz, N., \& Weinberg, M. D. 2003, ApJS, 149, 289

Bennert, N., Treu, T., Woo, J.-H., Malkan, M. A., Le Bris, A., Auger, M. W., Gallagher, S., \& Blandford, R. D. 2009, ApJ, submitted

Bentz, M. C., Peterson, B. M., Netzer, H., Pogge, R. W., \& Vestergaard, M. 2009, ApJ, 697, 160

Bentz, M. C., Peterson, B. M., Pogge, R. W., Vestergaard, M., \& Onken, C. A. 2006, ApJ, 644, 133

Bianchi, S., Guainazzi, M., Matt, G., \& Fonseca Bonilla, N. 2007, A\&A, 467, L19

Bolzonella, M., et al. 2009, A\&A, in press (arXiv:0907.0013)

Bondi, M., Ciliegi, P., Schinnerer, E., Smolčić, V., Jahnke, K., Carilli, C., \& Zamorani, G. 2008, ApJ, 681, 1129

Bongiorno, A., et al. 2007, A\&A, 472, 443

Boroson, T. A., \& Green, R. F. 1992, ApJS, 80, 109

Botte, V., Ciroi, S., Rafanelli, P., \& Di Mille, F. 2004, AJ, 127, 3168

Bower, R. G., Benson, A. J., Malbon, R., Helly, J. C., Frenk, C. S., Baugh, C. M., Cole, S., \& Lacey, C. G. 2006, MNRAS, 370, 645

Boyle, B. J., Shanks, T., Croom, S. M., Smith, R. J., Miller, L., Loaring, N., \& Heymans, C. 2000, MNRAS, 317, 1014

Bruhweiler, F., \& Verner, E. 2008, ApJ, 675, 83

Brusa, M., et al. 2009, A\&A, in press (arXiv:0910.1007)

Bruzual, G., \& Charlot, S. 2003, MNRAS, 344, 1000 (BC03)

Calzetti, D., et al. 2000, ApJ, 533, 682

Capak, P., et al. 2007, ApJS, 172, 99

Cavaliere, A., \& Vittorini, V. 2002, ApJ, 570, 114

Chabrier, G. 2002, ApJ, 567, 304

Cimatti, A., et al. 2008, A\&A, 482, 21

Ciotti, L., \& Ostriker, J. P. 1997, ApJ, 487, L105

Ciotti, L., \& Ostriker, J. P. 2001, ApJ, 551, 131

Coldberg, J. M., \& Di Matteo, T. 2008, MNRAS, 387, 1163

Croton, D. J. 2006, MNRAS, 369, 1808

Croton, D. J., et al. 2006, MNRAS, 365, 11

Decarli, R., Falomo, R., Treves, A., Labita, M., Kotilainen, J. K., \& Scarpa, R. 2009, MNRAS, in press (arXiv:0911.2988)

Di Matteo, T., Colberg, J., Springel, V., Hernquist, L., \& Sijacki, D. 2008, ApJ, 676, 33

Di Matteo, T., Croft, R. A., Springel, V., \& Hernquist, L. 2003, ApJ, 593, 56

Di Matteo, T., Springel, V., \& Hernquist, L. 2005, Nature, 433, 604

Elvis, M., et al. 2004, ApJS, 95, 1

Elvis, M., et al. 2009, ApJS, 184, 158

Fabian, A. C. 1999, MNRAS, 308, L39

Ferrarese, L., \& Merritt, D. 2000, ApJ, 539, L9

Fine, S., et al. 2006, MNRAS, 373, 613

Fioc, M., \& Rocca-Volmerange, B. 1997, A\&A, 326, 950

Fontana, A., et al. 2004, A\&A, 459, 745

Fontanot, F., Monaco, P., Cristiani, S., \& Tozzi, P. 2006, MNRAS, 373, 1173

Francis, P. J. 1993, ApJ, 407, 519

Gavignaud, I., et al. 2008, A\&A, 492, 637

Gebhardt, K., et al. 2000, ApJ, 539, L13

Graham, A. 2004, ApJ, 613, L33

Graham, A. W. 2007, MNRAS, 379, 711

Granato, G. L., Silva, L., Monaco, P., Panuzzo, P., Salucci, P., De Zotti, G., \& Danese, L. 2001, MNRAS, 324, 757

Granato, G. L., De Zotti, G., Silva, L., Bressan, A., \& Danese, L. 2004, ApJ, 600,580

Greene, J. E., \& Ho, L. C. 2005, ApJ, 627, 721

Greene, J. E., \& Ho, L. C. 2006, ApJ, 641, 117

Gültekin, K., et al. 2009, ApJ, 698, 1986

Häring, N., \& Rix, H.-W. 2004, ApJ, 604, L89

Hickox, R. C., et al. 2009, ApJ, 696, 891

Ho, L. C. 2007, ApJ, 669, 821

Ho, L. C., Darling, J., \& Greene, J. E. 2008, ApJ, 681, 128

Hopkins, P. F., \& Hernquist, L. 2009, ApJ, 698, 1550

Hopkins, P. F., Hernquist, L., Cox, T. J., Di Matteo, T., Robertson, B., \& Springel, V. 2006a, ApJS, 163, 1

Hopkins, P. F., Hernquist, L., Cox, T. J., Keres, D., \& Wuyts, S. 2009, ApJ, 691 , 1424 
Hopkins, P. F., Hernquist, L., Cox, T. J., Robertson, B., \& Krause, E. 2007a, ApJ, 669,67

Hopkins, P. F., Richards, G. T., \& Hernquist, L. 2007b, ApJ, 654, 731

Hopkins, P. F., Robertson, B., Krause, E., Hernquist, L., \& Cox, T. J. 2006b, ApJ, 652,107

Ilbert, O., et al. 2009, ApJ, in press (arXiv:0903.0102)

Jahnke, K., Kuhlbrodt, B., \& Wisotzki, L. 2004, MNRAS, 352, 399

Jahnke, K., et al. 2009, ApJ, 706, L215

Johansson, P. H., Naab, T., \& Burkert, A. 2009, ApJ, 690, 802

Kaspi, S., Smith, P. S., Netzer, H., Maoz, D., Jannuzzi, B. T., \& Giveon, U. 2000, ApJ, 533, 631

Kauffmann, G., \& Haehnelt, M. 2000, MNRAS, 311, 576

Kauffmann, G., et al. 2003, MNRAS, 346, 1055

Kellerman, K. I. 1964, ApJ, 140, 969

Kim, M., Ho, L. C., Peng, C. Y., Barth, A. J., Im, M., Martini, P., \& Nelson, C. H. 2008, ApJ, 687, 767

Koekemoer, A. M., et al. 2007, ApJS, 172, 196

Kollmeier, J., et al. 2006, ApJ, 648, 128

Komossa, S., \& Xu, D. 2007, ApJ, 667, L33

Kormendy, J., \& Bender, R. 2009, ApJ, 691, L142

Kormendy, J., \& Gebhardt, K. 2001, in AIP Conf. Proc. 586, Supermassive Black Holes in Galactic Nuclei, ed. H. Martel \& J. C. Wheeler (Melville, NY: AIP), 363

Le Floch, E., et al. 2009, ApJ, 703, 222

Letawe, G., Magain, P., Courbin, F., Jablonka, P., Jahnke, K., Meylan, G., \& Wisotzki, L. 2007, MNRAS, 378, 83

Lamastra, A., Menci, N., Maiolino, R., Fiore, F., \& Merloni, A. 2009, MNRAS, submitted

Lauer, T. R., Tremaine, S., Richstone, D., \& Faber, S. M. 2007, ApJ, 670, 249

Lilly, S. J., et al. 2007, ApJS, 172, 70

Lilly, S. J., et al. 2009, ApJS, 184, 218

Longhetti, M., \& Saracco, P. 2009, MNRAS, 394, 774

Madau, P., Pozzetti, L., \& Dickinson, M. 1998, ApJ, 498, 106

Magorrian, J., et al. 1998, AJ, 115, 2285

Malbon, R. K., Baugh, C. M., Frenk, C. S., \& Lacey, C. G. 2007, MNRAS, 382, 1394

Malmquist, K. G. 1924, Medd. Lund Astron. Obs. II, 32, 64

Maraston, C. 2005, MNRAS, 362, 799

Marconi, A., Axon, D., Maiolino, R., Nagao, T., Pastorini, G., Pietrini, P., Robinson, A., \& Torricelli, G. 2008, ApJ, 678, 693

Marconi, A., \& Hunt, L. K. 2003, ApJ, 589, L21

Marconi, A., Risaliti, G., Gilli, R., Hunt, L. K., Maiolino, R., \& Salvati, M. 2004, MNRAS, 351, 169

Marulli, F., Bonoli, S., Branchini, E., Moscardini, L., \& Springel, V. 2008, MNRAS, 385, 1846

Mc Cracken, H. J., et al. 2009, ApJ, submitted (arXiv:0910.2705)

McGill, K. L., et al. 2008, ApJ, 673, 703

McLure, R. J., \& Dunlop, J. S. 2001, MNRAS, 327, 199

McLure, R. J., \& Dunlop, J. S. 2004, MNRAS, 352, 1390

McLure, R. J., \& Jarvis, M. J. 2002, MNRAS, 337, 109

Menci, N., Fontana, A., Giallongo, E., Grazian, A., \& Salimbeni, S. 2006, ApJ, 647,753

Merloni, A., \& Heinz, S. 2008, MNRAS, 388, 1011

Merloni, A., Rudnick, G., \& Di Matteo, T. 2004, MNRAS, 354, L37

Monaco, G., Salucci, P., \& Danese, L. 2000, MNRAS, 311, 279

Netzer, H., Lira, P., Trakhtenbrot, B., Shemmer, O., \& Cury, I. 2007, ApJ, 671, 1256

Novak, G. S., Faber, S. M., \& Dekel, A. 2006, ApJ, 637, 96

Onken, C. A., Ferrarese, L., Merritt, D., Peterson, B. M., Pogge, R. W., Vestergaard, M., \& Wandel, A. 2004, ApJ, 615, 645
Peng, C. Y., Impey, C. D., Ho, L. C., Barton, E. H., \& Rix, H.-W. 2006a, ApJ, 640, 114

Peng, C. Y., Impey, C. D., Rix, H.-W., Kochanek, C. S., Keeton, C. R., Falco, E. E., Lehár, J., \& McLeod, B. A. 2006b, ApJ, 649, 616

Peterson, B. M., et al. 2004, ApJ, 613, 682

Polletta, M., et al. 2007, ApJ, 663, 81 (P07)

Pozzetti, L., et al. 2007, A\&A, 474, 443

Prevot, M. L., Lequeux, J., Prevot, L., Maurice, E., \& Rocca-Volmerange, B. 1984, A\&A, 132, 389

Richards, G., et al. 2006, ApJS, 166, 470

Robertson, B., Hernquist, L., Cox, T. J., Di Matteo, T., Hopkins, P. F., Martini, P., \& Springel, V. 2006, ApJ, 641, 90

Ross, N. P., Assef, R. J., Kochanek, C. S., Falco, E., \& Poindexter, S. D. 2009, ApJ, 702, 472

Salpeter, E. E. 1955, ApJ, 121, 161

Salvato, M., et al. 2009, ApJ, 690, 1250

Salviander, S., Shields, G. A., Gebhardt, K., \& Bonning, E. W. 2007, ApJ, 662 131

Sánchez, S. F., et al. 2004, ApJ, 614, 589

Sanders, D. B., et al. 2007, ApJS, 172, 86

Schinnerer, E., et al. 2007, ApJS, 172, 46

Schramm, M., Wisotzki, L., \& Jahnke, K. 2008, A\&A, 478, 311

Scoville, N., et al. 2007, ApJS, 172, 1

Shankar, F., Bernardi, M., \& Haiman, Z. 2009, ApJ, 694, 867

Shankar, F., Salucci, P., Granato, G. L., De Zotti, G., \& Danese, L. 2004, MNRAS, 354, 1020

Shapiro, K. L., et al. 2009, ApJ, 701, 955

Shen, J., Vanden Berk, D. E., Schneider, D. P., \& Hall, P. B. 2008, AJ, 135, 928

Shields, G. A., Gebhardt, K., Salviander, S., Wills, B. J., Xie, B., Brotherton, M. S., Yuan, J., \& Dietrich, M. 2003, ApJ, 583, 124

Shields, G. A., Menezes, K. L., Massart, C. A., \& Vanden Bout, P. 2006, ApJ, 641,683

Sijacki, D., Springel, V., Di Matteo, T., \& Hernquist, L. 2007, MNRAS, 380, 877

Silk, J., \& Rees, M. J. 1998, A\&A, 331, L1

Silva, L., Granato, G. L., Bressan, A., \& Danese, L. 1998, ApJ, 509, 103

Silverman, J. D., et al. 2008, ApJ, 675, 1025

Silverman, J. D., et al. 2009, ApJ, 696, 396

Sołtan, A. 1982, MNRAS, 200, 115

Somerville, R. S. 2009, MNRAS, 399, 1988

Somerville, R. S., Hopkins, P. F., Cox, T. J., Robertson, B. E., \& Hernquist, L. 2008, MNRAS, 391, 481

Springel, V., Di Matteo, T., \& Hernquist, L. 2005, ApJ, 620, L79

Surace, et al. 2005, SWIRE Data Release 2 (Pasadena, CA: CalTech), http://swire.ipac.caltech.edu

Taniguchi, Y., et al. 2007, ApJS, 172, 9

Tremaine, S., et al. 2002, ApJ, 574, 554

Treu, T., Woo, J.-H., Malkan, M. A., \& Blandford, R. D. 2007, ApJ, 667, 117

Trump, J., et al. 2009, ApJ, 700, 49

Vestergaard, M., \& Peterson, B. M. 2006, ApJ, 641, 689

Vestergaard, M., \& Wilkes, B. J. 2001, ApJS, 134, 1

Volonteri, M., Haardt, F., \& Madau, P. 2003, ApJ, 582, 559

Walter, F., et al. 2004, ApJ, 615, L17

Wandel, A., Peterson, B. M., \& Malkan, M. A. 1999, ApJ, 526, 579

Wyithe, J. S. B., \& Loeb, A. 2003, ApJ, 595, 614

Woo, J.-H., Treu, T., Malkan, M. A., \& Blandford, R. D. 2006, ApJ, 645, 900

Woo, J.-H., Treu, T., Malkan, M. A., \& Blandford, R. D. 2008, ApJ, 681, 925 